ARTICLE

\title{
Distinct axial and lateral interactions within homologous filaments dictate the signaling specificity and order of the AIM2-ASC inflammasome
}

Mariusz Matyszewski id 1,3, Weili Zheng (D 2 , Jacob Lueck', Zachary Mazanek¹, Naveen Mohideen', Albert Y. Lau ${ }^{1}$, Edward H. Egelman (1) ${ }^{2} \&$ Jungsan Sohn (10) ${ }^{1 凶}$

Inflammasomes are filamentous signaling platforms integral to innate immunity. Currently, little is known about how these structurally similar filaments recognize and distinguish one another. A cryo-EM structure of the AIM2 ${ }^{\text {PYD }}$ filament reveals that the architecture of the upstream filament is essentially identical to that of the adaptor ASC ${ }^{\text {PYD }}$ filament. In silico simulations using Rosetta and molecular dynamics followed by biochemical and cellular experiments consistently demonstrate that individual filaments assemble bidirectionally. By contrast, the recognition between AIM2 and ASC requires at least one to be oligomeric and occurs in a head-to-tail manner. Using in silico mutagenesis as a guide, we also identify specific axial and lateral interfaces that dictate the recognition and distinction between AIM2 and ASC filaments. Together, the results here provide a robust framework for delineating the signaling specificity and order of inflammasomes.

\footnotetext{
${ }^{1}$ Department of Biophysics and Biophysical Chemistry, Johns Hopkins University School of Medicine, Baltimore, MD, USA. ${ }^{2}$ Department of Biochemistry and Molecular Genetics, University of Virginia School of Medicine, Charlottesville, VA, USA. ${ }^{3}$ Present address: Department of Cellular and Molecular Medicine, University of California San Diego, La Jolla, CA, USA. ${ }^{凶}$ email: jsohn@jhmi.edu
} 
nflammasomes are filamentous signaling platforms and play key roles in the metazoan innate immune system ${ }^{1}$. These supra-structures assemble upon detecting molecular signatures arising from various intracellular catastrophes, such as genomic instability, dysfunctional organelles, and pathogen invasion ${ }^{1}$. Mammals have at least 15 different receptors that lead to the assembly of inflammasomes whose ultimate goal is to induce the polymerization of procaspase-1, activating the zymogen protease by proximity-induced autoproteolysis ${ }^{1}$. Caspase- 1 then executes two key innate immune responses: the cleavage/maturation of pro-inflammatory cytokines, such as interleukin- $1 \beta$ and -18 , and the initiation of pyroptosis ${ }^{1}$. Inflammasomes play essential roles in host defense against pathogen invasion (e.g., coronaviruses, herpesviridae, and Listeria monocytogene $)^{1-6}$. In addition, malfunctioning inflammasomes promote acute and chronic autoinflammatory diseases (e.g., severe COVID-19, rheumatoid arthritis, and systemic lupus erythematosus (SLE) $)^{7-9}$, metabolic disorders (type 2 diabetes) ${ }^{10,11}$, and even tumorigenesis (colon cancer, lung cancer, and oral cancer) $)^{12,13}$

Inflammasome receptors contain multiple functional domains for autoinhibition, signal recognition, and oligomerization ${ }^{1,14}$. Importantly, the N-terminal pyrin domain (PYD) acts as the primary signal transduction module in the vast majority of inflammasomes ${ }^{1,14,15}$. PYDs are six-helix bundles that belong to the death-domain (DD) superfamily and can assemble into helical filaments. For instance, incoming signals such as viral nucleic acids induce the assembly of a receptor PYD filament ${ }^{14,16-18}$. The upstream PYD filaments then nucleate the filamentation of the PYD of central adaptor ASC (ASC $\left.{ }^{\text {PYD }}\right)^{14,18-20}$, leading to the oligomerization of the CARD of ASC (ASCCARD) to recruit and trigger the polymerization (activation) of procaspase-1 (ASC: apoptosis-associated speck-forming protein containing caspaserecruiting domain (CARD); CARDs are also six-helix bundles that belong to the DD family) ${ }^{14,18,21,22}$.

Although the structural mechanisms by which inflammasomes assemble are increasingly better understood ${ }^{14,17-25}$, little is known about the mechanisms that direct the signaling order (sequence) and specificity. For instance, all published cryoelectron microscopy (cryo-EM) structures of PYD filaments show essentially the same helical architectures (six subunits per helical turn $)^{18,20}$; all CARD filaments also show the same helical architectures (four subunits per helical turn) $14,21,23$. These observations then led to a well-accepted model, in which the architectural complementarity between upstream and downstream filaments underpins the recognition $14,17,18,20,21,26$. However, it raises a considerably more complex problem as to how these similar helical filaments built from homologous protomers distinguish and recognize one another within respective subfamilies. Here, we address this fundamental mechanistic issue in the cytosolic double-stranded (ds)DNA-sensing AIM2-ASC inflammasome ${ }^{27}$. AIM2 (absent in melanoma 2) is a bipartite protein composed of the N-terminal PYD followed by the dsDNA-binding HIN domain (hematopoietic interferon-inducible nuclear antigen). Upon binding cytosolic dsDNA via its HIN domain, AIM2 ${ }^{\mathrm{PYD}}$ assembles into filaments, inducing the polymerization of $\mathrm{ASC}^{12,16,17,19}$. AIM2 is essential for the host defense against numerous pathogenic viruses and bacteria ${ }^{6,12,16,27-30}$. AIM2 also plays vital roles in neuronal development by regulating timely cell death $^{31}$. However, dysregulated AIM2 leads to various maladies, such as SLE, chronic kidney diseases, and lung cancer ${ }^{12,27,32-34}$.

We present a cryo-EM structure of the AIM2 $2^{\mathrm{PYD}}$ filament at $3.2 \AA$ resolution, which reveals that its architecture is indeed identical to that of the ASC ${ }^{\mathrm{PYD}}$ filament. Using our structure, we then investigate how $\mathrm{AIM} 2^{\mathrm{PYD}}$ and $\mathrm{ASC} \mathrm{PYD}^{\mathrm{P}}$ filaments recognize, and distinguish each other by Rosetta and molecular dynamics (MD) simulations. Our in silico analyses consistently suggest that the energy landscapes that underpin the assembly of individual filaments do not impose directionality. By contrast, the energy landscape that governs the recognition between $\mathrm{AIM} 2^{\mathrm{PYD}}$ and $\mathrm{ASC}^{\mathrm{PYD}}$ is polarized in a head-to-tail manner. Multiple biochemical experiments corroborate that individual filaments assemble bidirectionally. Moreover, $\mathrm{AIM} 2^{\mathrm{PYD}}$ and $\mathrm{ASC} \mathrm{CYD}^{\mathrm{P}}$ filaments do not co-assemble, and the signal transduction from AIM2 to ASC occurs unidirectionally only when at least one is oligomeric. Using Rosetta-based in silico mutagenesis as a guide, our biochemical and cellular experiments consistently show that lateral interfaces of AIM $2^{\text {PYD }}$ drive its bidirectional assembly. We also identify specific axial interfaces that mediate the recognition between AIM2 ${ }^{\mathrm{PYD}}$ and ASC ${ }^{\mathrm{PYD}}$. Together, we demonstrate that distinct interfaces within homologous filaments direct signaling order and specificity of inflammasomes. We also set forth a broadly applicable multidisciplinary platform for delineating the signal transduction order, specificity, and directionality of filamentous assemblies.

\section{Results}

The cryo-EM structure of AIM2 ${ }^{\text {PYD }}$. Using EM of negatively stained samples (nsEM), we previously found that the helical symmetry of the AIM2 $2^{\text {PYD }}$ filament is consistent with that of the ASC $^{\mathrm{PYD}}$ filament ${ }^{17}$, and thus proposed that architectural complementarity is important for their recognition. However, the published high-resolution cryo-EM structure of the AIM2 ${ }^{\mathrm{PYD}}$ filament displays an altered helical architecture because the $\mathrm{N}$ terminal green fluorescence protein (GFP)-tag interferes with assembly ${ }^{35}$. Thus, we first determined the cryo-EM structure of the AIM2 2 PYD filament using an untagged recombinant protein.

Cryo-EM images showed that AIM2 ${ }^{\mathrm{PYD}}$ filaments are straight helical rods (Fig. 1A). The average power spectrum of 512-pixellong nonoverlapping filament segments showed that the AIM2 ${ }^{\text {PYD }}$ filament displays a six-start, C3 helical symmetry of $54.4^{\circ}$ rotation ( $\sim 6$ subunits per helical turn) and an axial rise of $14 \AA$ (Fig. 1B). These parameters are remarkably similar to those of the ASC ${ }^{\mathrm{PYD}}$ filament ${ }^{18}$, further solidifying the concept that the upstream receptors provide structural templates for downstream assemblies in inflammasomes ${ }^{14,17,26}$. We fit the crystal structure of AIM2 $2^{\text {PYD }}$ into the EM map for initial modeling ${ }^{36}$, and the refined high-resolution map allowed us to model in most bulky and aliphatic side chains (Fig. 1C). The resolution of the final model was $3.2 \AA$ according to the gold standard method (Supplementary Fig. 1A). The diameter of the outer rim is $\sim 94 \AA$ and that of the inner cavity is $\sim 25 \AA$ (Fig. 1D). The structure of individual AIM2 ${ }^{\mathrm{PYD}}$ protomers is identical to the crystal structure of AIM2 ${ }^{\mathrm{PYD}}$ monomer (Supplementary Fig. 1B), thus indicating that, unlike the PYD of NLRP6 (ref. ${ }^{20}$ ), an AIM2 $2^{\mathrm{PYD}}$ monomer does not undergo any conformational changes during activation. As seen from the ASCPYD filament, each $\mathrm{AIM} 2{ }^{\mathrm{PYD}}$ subunit contributes three unique protein-protein interaction interfaces (Fig. 1E). The type 1a:1b interface is largely composed of side-chain interactions, while the type $2 \mathrm{a}: 2 \mathrm{~b}$ and type $3 \mathrm{a}: 3 \mathrm{~b}$ interfaces involved both side-chain and backbone interactions (Fig. 1E). We also noted several side chains previously implicated in filament assembly throughout different interfaces (e.g., L11, D19, F27, and I46; Fig. 1E) ${ }^{17}$. Aligning the new AIM2PYD filament to the GFP-AIM2 ${ }^{\text {PYD }}$ filament demonstrates that although the lateral interactions are largely conserved, the axial positions are significantly different due to the altered helical symmetry (five subunits per turn in the GFP-tagged filament vs. six subunits per turn in the untagged filament; Supplementary Fig. 1C). On the other hand, aligning the cryo-EM structures of AIM2 ${ }^{\mathrm{PYD}}$ and ASC ${ }^{\mathrm{PYD}}$ filaments demonstrates their congruent architectures (Fig. 1F). The subtle difference in subunit 


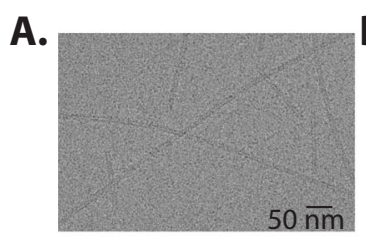

B.

D.

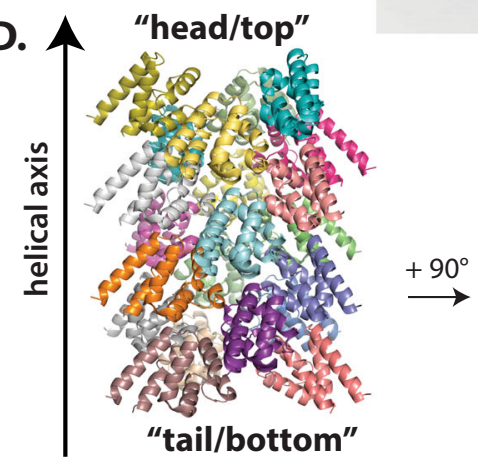

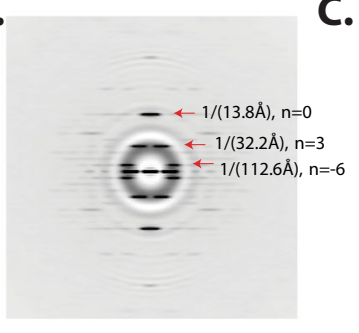

C.

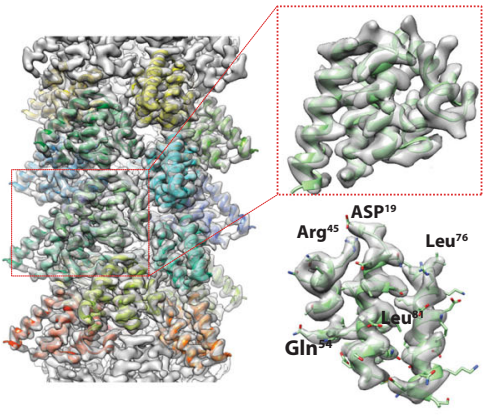

tail (botton)

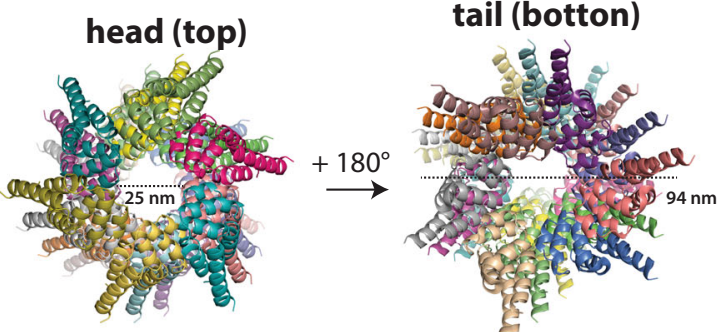

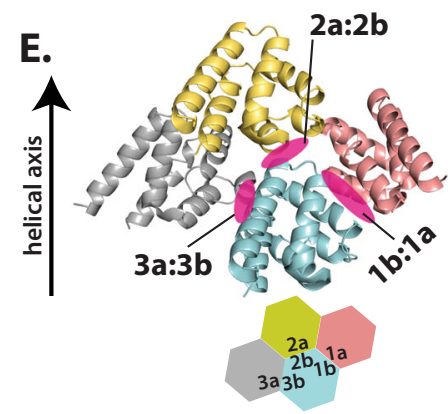
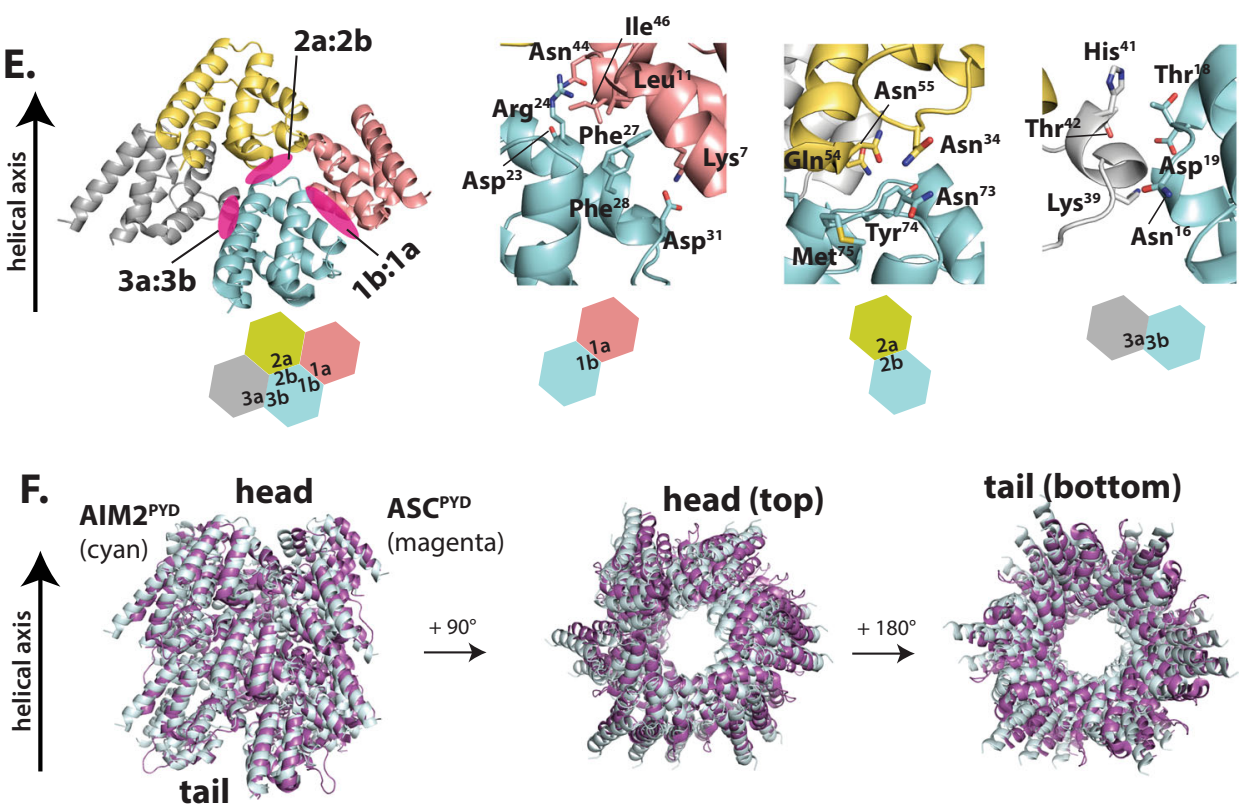

Fig. 1 AIM2 ${ }^{\text {PYD }}$ assembles into an architecturally congruent filament as the ASCPYD filament. A A sample cryo-electron micrograph of the AIM2PYD filament (total 976 micrographs were taken). B An average power spectrum of the AIM2 ${ }^{\text {PYD }}$ filament from 512 px-long nonoverlapping segments. C The AIM2 ${ }^{\text {PYD }}$ filament model built into the EM map. A subunit with visible side chains is shown. D The model of AIM2 ${ }^{P Y D}$ filament. Each subunit is colored differently. E A cartoon representation of three unique filament interface types. Side chains at each filament interface are shown as a stick configuration. F Overlays between AIM2 ${ }^{\mathrm{PYD}}$ and $\mathrm{ASC} \mathrm{PYD}^{\mathrm{P}}$ (PDB ID: 3J63) filaments.

positions between $\mathrm{AIM} 2^{\mathrm{PYD}}$ and $\mathrm{ASC} \mathrm{PYD}^{\mathrm{PD}}$ filaments along the helical axis could reflect the unique side-chain interactions that mediate their respective filament assembly or the inherent flexibility of biomolecular structures (Fig. 1F). Nevertheless, the near perfect architectural complementarity between AIM2 ${ }^{\text {PYD }}$ and ASC ${ }^{\text {PYD }}$ filaments supports the idea that upstream filaments provide structural templates for the assembly of downstream filaments ${ }^{14,17,26,37}$.

Deciphering the specificity and directionality of the AIM2-ASC inflammasome using Rosetta and MD. AIM2 $2^{\mathrm{PYD}}$ and $\mathrm{ASC} \mathrm{C}^{\mathrm{PYD}}$ monomers are homologous and structurally highly conserved (root-mean-squared-deviation, RMSD 0.5 $\mathrm{A}$ ), and our new cryoEM structure shows that they indeed assemble into essentially identical filaments (Fig. 1F). These observations raise significantly more complex questions as to whether and how these suprastructures distinguish and recognize each other. Importantly, such questions are germane to all filamentous signaling platforms employing PYDs or CARDs $21,22,26,37-39$. Thus, to establish a broadly relevant method for tackling these questions, we employed a computational approach using Rosetta. First, we tested whether RosettaDock ${ }^{40}$ could recapitulate the cryo-EM structures by docking an $\mathrm{AIM} 2^{\mathrm{PYD}}$ monomer into our AIM2 ${ }^{\mathrm{PYD}}$ filament structure (also an $\mathrm{ASC}^{\mathrm{PYD}}$ monomer to the $\mathrm{ASC}^{\mathrm{PYD}}$ filament (PDB ID: 3J63) ${ }^{18}$ ). For instance, each PYD protomer provides three unique interfaces in $\mathrm{AIM} 2^{\mathrm{PYD}}$ and $\mathrm{ASC} \mathrm{C}^{\mathrm{PYD}}$ filaments (i.e., six distinct surfaces; Figs. $1 \mathrm{E}$ and $2 \mathrm{~A}$ ). To facilitate docking experiments, we generated a honeycomb-like side view of $\mathrm{AIM} 2^{\mathrm{PYD}}$ and $\mathrm{ASC}{ }^{\mathrm{PYD}}$ filaments, in which the center protomer 

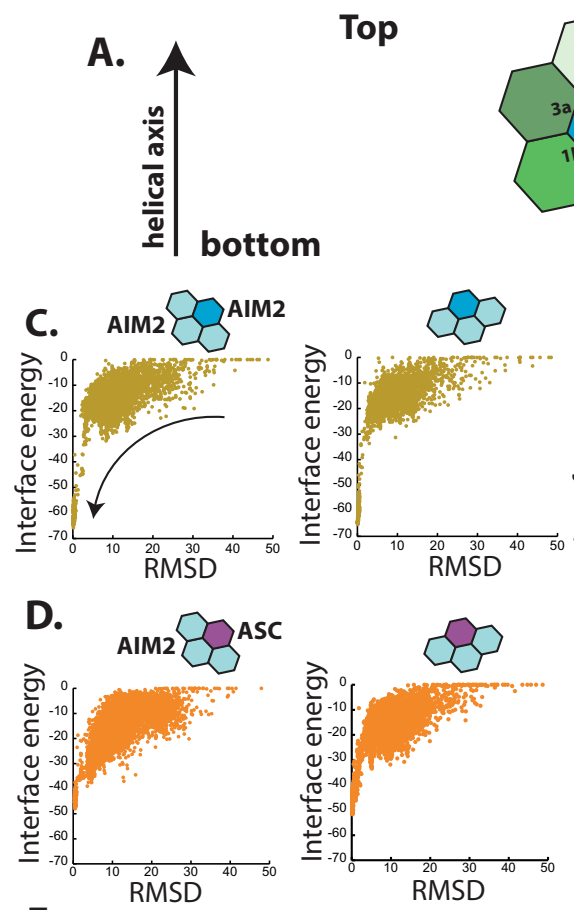

E.
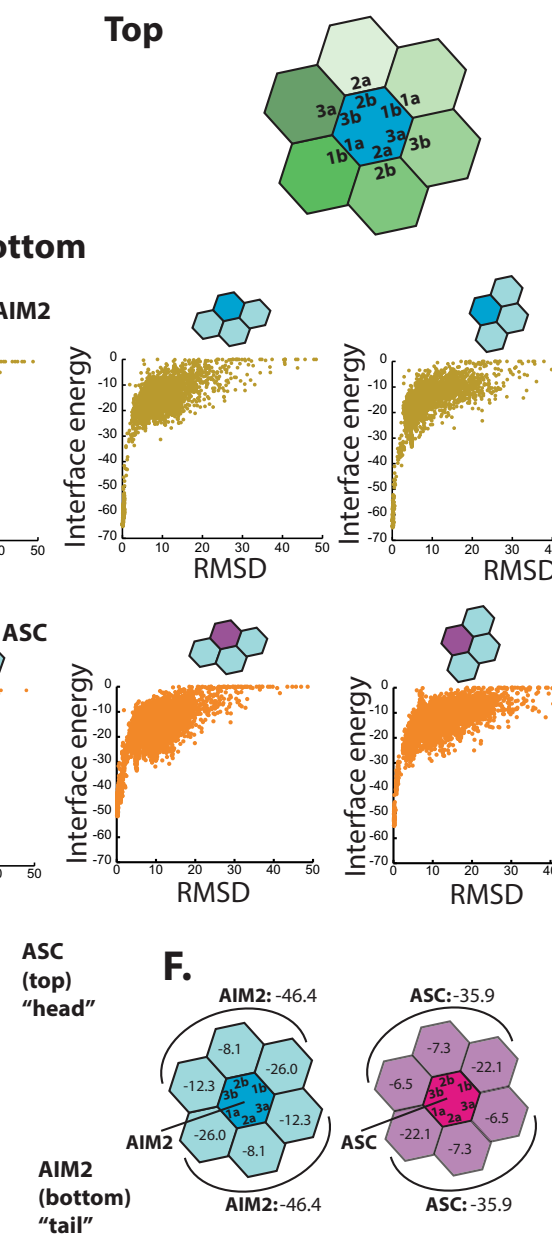
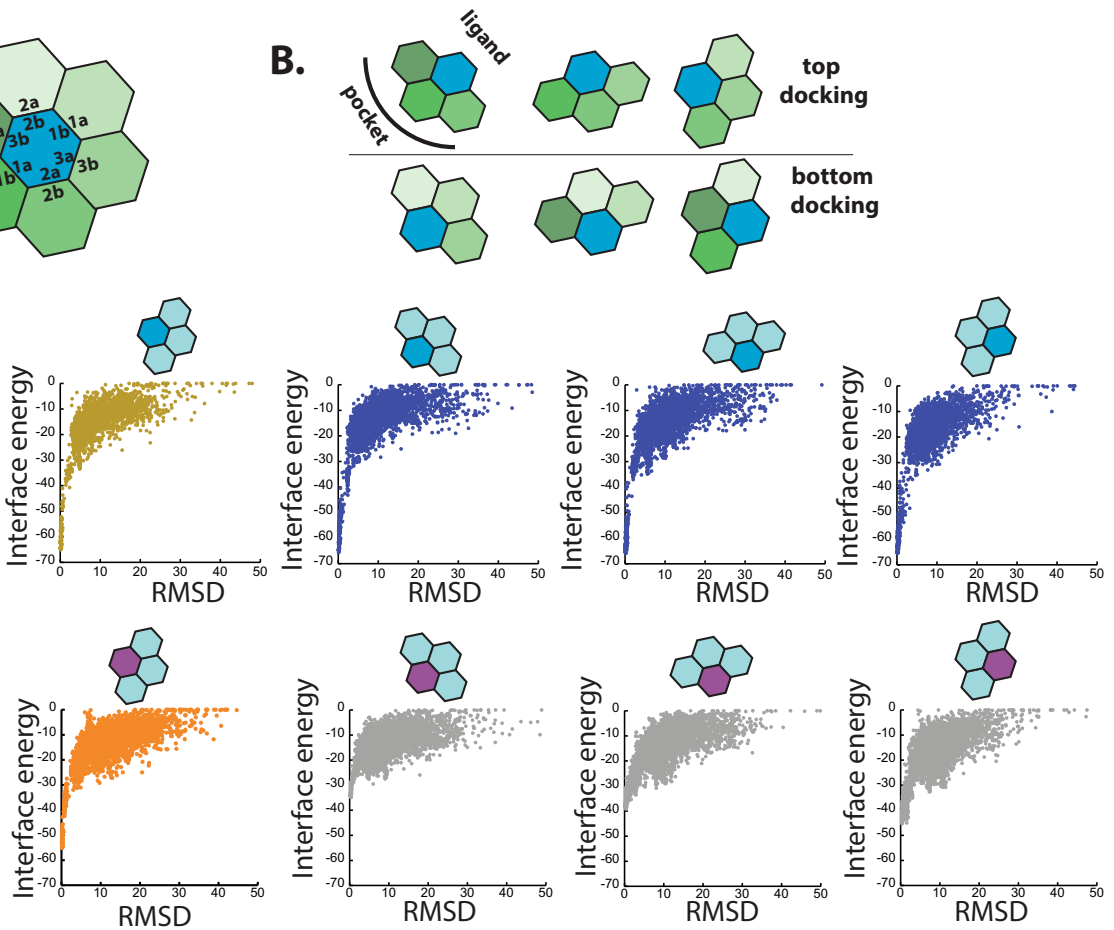

G.
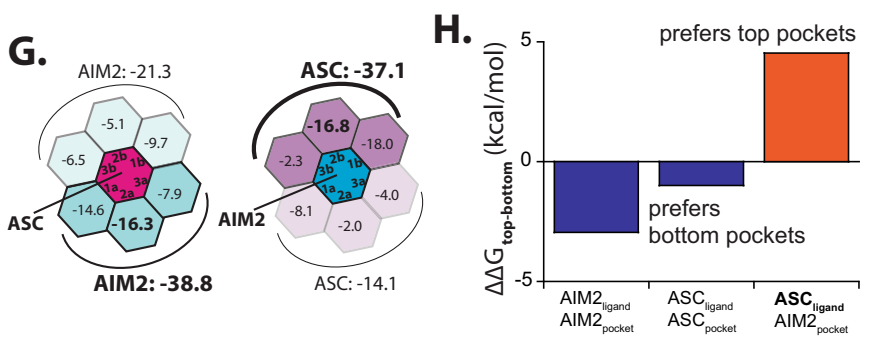

Fig. 2 In silico studies suggest that homotypic filaments assemble bidirectionally and the recognition between AIM2 and ASC occurs unidirectionally. A Cartoon representations of honeycombs. Each interface type is labeled in the hexagons which represent PYD monomers. B Rosetta docking strategy. Top docking indicates that a ligand monomer docks onto the top surface of the pocket, while bottom docking is the opposite. C Plots of Rosetta interface energy scores vs. RMSD for top and bottom docking results for AIM2 ${ }^{\mathrm{PYD}}$ assembly. The B represents simulations conducted in C. D Plots of Rosetta interface energy scores vs. RMSD for docking an ASCPYD monomer on the top or bottom of AIM2PYD pockets. $\mathbf{E}$ A model of the AIM2 ${ }^{P Y D}$ filament recognition of the $A S C^{P Y D}$ filament. F, G Rosetta interface energy scores at individual filament interfaces for homotypic and heterotypic assemblies. AIM2 ${ }^{P Y D}$-AIM2 ${ }^{P Y D}$, $A S C^{P Y D}-A S C^{P Y D}, A S C^{P Y D}-A I M 2^{P Y D}$, and $A I M 2^{P Y D}-A S C^{P Y D}$. Each hexagon represents $A I M 2^{P Y D}$ or $A S C^{P Y D}$ monomer. $\mathbf{H} A$ plot for the difference in free energy $(\Delta \Delta \mathrm{G})$ for dissociating ligand PYDs from the top or bottom pockets.

makes all six possible contacts (Fig. 2A). We then divided the honeycomb into six unique subsections consisting of one ligand docked into a pocket created by three adjacent subunits (Fig. 2B). Using the local docking method in Rosetta ${ }^{40}$, we performed 5000 independent docking simulations between a ligand-pocket pair from each subsection, then compared the interface energy and RMSD from the cryo-EM structures.

For each filament, both parameters decreased concurrently, while displaying uniform energy scores from all subsections (Fig. 2C (arrow) and Supplementary Fig. 2A), indicating that RosettaDock can recapitulate the cryo-EM structures. The more favorable energy scores from the AIM2 ${ }^{\text {PYD }}$ filament suggest that it is is more stable than the ASC ${ }^{\text {PYD }}$ filament (typically $-70 \mathrm{~s}$ for $\mathrm{AIM}^{2}{ }^{\mathrm{PYD}}$ complexes vs. $-50 \mathrm{~s}$ for $\mathrm{ASC}^{\mathrm{PYD}}$ complexes). Importantly, the uniform energy scores throughout the top and bottom subsections (Fig. 2C and Supplementary Fig. 2A) suggest that individual filaments would assemble bidirectionally. Next, we docked an $\mathrm{ASC}^{\mathrm{PYD}}$ monomer (ligand) onto all six pockets of the $\mathrm{AIM} 2^{\mathrm{PYD}}$ filament and vice versa (Fig. $2 \mathrm{D}$ and Supplementary Fig. 2B). We first noted that the interface energies are not as favorable as the $\mathrm{AIM} 2^{\mathrm{PYD}} \cdot \mathrm{AIM}_{2}{ }^{\mathrm{PYD}}$ complexes $(-60$ or worse for $\mathrm{ASC}^{\mathrm{PYD}} \cdot \mathrm{AIM}_{2}{ }^{\mathrm{PYD}}$ complxes; Fig. $2 \mathrm{D}$ and Supplementary Fig. 2B). Moreover, docking ASC ${ }^{\mathrm{PYD}}$ on the top pockets of the AIM2 ${ }^{\text {PYD }}$ filament was significantly more favorable than docking at the bottom. (Fig. 2D orange vs. gray). Docking AIM2 ${ }^{\mathrm{PYD}}$ on the $A C^{P Y D}$ filament also showed that $\mathrm{AIM} 2^{\mathrm{PYD}}$ prefers the bottom half of the ASC ${ }^{\mathrm{PYD}}$ filament with the energy scores as favorable as the homotypic $\mathrm{ASC}^{\mathrm{PYD}}$ assembly (Supplementary Fig. $2 \mathrm{~A}, \mathrm{~B}$ ( $2 \mathrm{~B}$ orange vs. gray)). These results suggest that individual filaments assemble bidirectionally, while the recognition between $\mathrm{AIM} 2^{\mathrm{PYD}}$ and $\mathrm{ASC} \mathrm{CPD}^{\mathrm{PYD}}$ occurs unidirectionally, where the top of the $\mathrm{AIM}_{2}{ }^{\mathrm{PYD}}$ filament recognizes the bottom of the ASC ${ }^{\text {PYD }}$ filament (Fig. 2E).

Next, we used Rosetta InterfaceAnalyzer ${ }^{41}$ to evaluate the interaction energies between homotypic and heterotypic interactions at the individual interfaces of the honeycomb (Fig. 2F). The $\mathrm{AIM} 2^{\mathrm{PYD}}$ complex also showed the most favorable overall interface energy scores (Fig. 2G). Moreover, for the respective homotypic assembly of $\mathrm{AIM} 2^{\mathrm{PYD}}$ and $\mathrm{ASC} \mathrm{CYD}^{\mathrm{PY}}$, the type 1 interface contributed most significantly with the top and bottom halves displaying symmetric energy scores (Fig. 2F). On the other hand, the interface energy scores were consistently worse, when 
A.

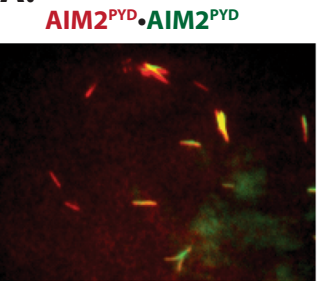

B.

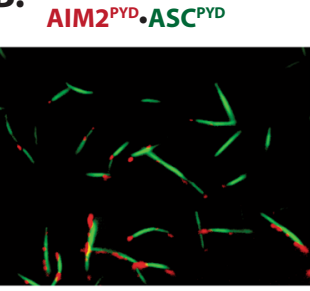
$\mathrm{ASC}^{\mathrm{PYD}} \cdot \mathrm{ASC} \mathrm{CPD}^{\mathrm{PYD}}$

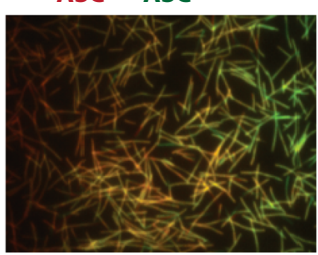

(AIM2 ${ }^{\text {PYD }}$ filament) + ASC ${ }^{\text {PYD }}$

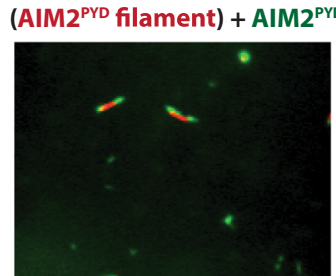

$\left(\right.$ ASC $^{\text {PYD }}$ filament $)+$ AIM2 $2^{\text {PY }}$

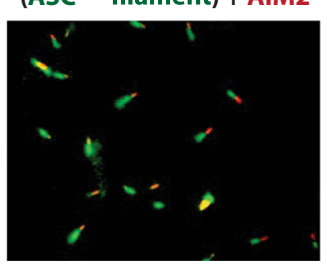

(ASC ${ }^{\text {PYD }}$ filament) + ASC $^{\text {PYD }}$
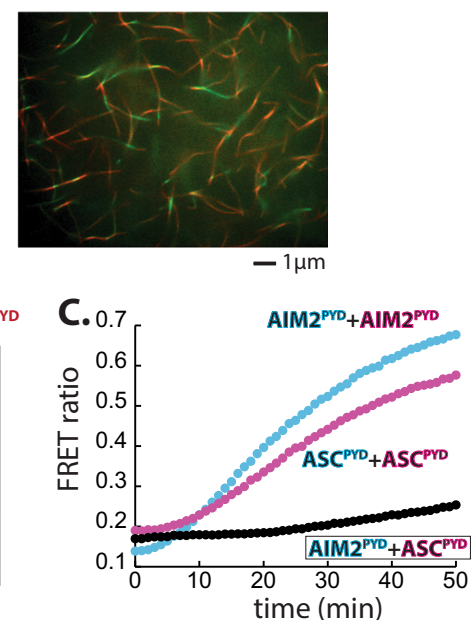

Fig. 3 Individual filaments assemble bidirectionally and the recognition between AIM2 and ASC occurs at a specific pole. A Fluorescent confocal microscopy images of Alexa $488^{-}$and Dylight $550^{-l a b e l e d ~ A I M 2 P Y D, ~ A l e x a ~} 488^{-}$and Dylight $550^{-l a b e l e d ~ A S C P Y D, ~ p r e a s s e m b l e d ~ A l e x a ~} 488^{-l a b e l e d ~ A I M 2 P Y D ~}$ filament and nascent Dylight ${ }_{550}$-labeled AIM2PYD, and preassembled Alexa $488^{-}$labeled ASC PYD filament and nascent Dylight $_{550}$-labeled ASC $^{\text {PYD }}$. B Fluorescent confocal microscopy images Alexa $488^{-l a b e l e d ~ A I M 2 P Y D}$ and Dylight ${ }_{550}$-labeled ASCPYD, preassembled Alexa $488^{-l a b e l e d}$ AIM2PYD filament and nascent Dylight 550 -labeled ASCPY, and preassembled Alexa $488^{\text {-labeled ASC }}{ }^{\text {PYD }}$ filament and nascent Dylight 550 -labeled AIM2 ${ }^{\text {PYD }}$. Images shown in $\mathbf{A}$ and $\mathbf{B}$ are representatives of at least three independent experiments. $\mathbf{C}$ The time-dependent changes in FRET ratios between donor or acceptor-labeled MBP-AIM2 ${ }^{\text {PYD }}$ and/or MBP-ASC ${ }^{P Y D}$ were monitored upon cleaving the MBP tag with TEVp.

$\mathrm{ASC}^{\mathrm{PYD}}$ was placed at the center of the AIM2 2 PYD honeycomb, except for the one between the type $2 \mathrm{a}$ of $\mathrm{ASC} \mathrm{CYD}^{\mathrm{PY}}$ and $2 \mathrm{~b}$ of AIM2 2 PYD (Fig. 2F vs. 2G). The overall energy scores between the top and bottom halves were again asymmetric, preferring a headto-tail-like direction, in which the top of $\mathrm{AIM}_{2} \mathrm{PYD}_{\mathrm{S}}$ favoring the bottom of ASCPYD and vice versa (Fig. 2E, G; the small difference in energy scores between Fig. 2F, G likely stemmed from the subtle architectural differences in two filaments).

To test whether the simulation results are not biased by a particular algorithm, we then used MD to calculate the free energy required to dissociate a ligand PYD from each pocket described in Fig. 2B (i.e., stability; Supplementary Fig. 2C, D). Consistent with the results from RosettaDock, MD simulations suggested the AIM2 ${ }^{\mathrm{PYD}}$ filament complex to be most stable, followed by $\mathrm{ASC}^{\mathrm{PYD}} \cdot \mathrm{AIM} 2^{\mathrm{PYD}}$ then $\mathrm{ASC}{ }^{\mathrm{PYD}}$ complexes. (Supplementary Fig. 3B; see also Supplementary Fig. 3C for images showing the dissociation of each monomer before and after the simulation). We compared the sum of energies required to dissociate a ligand PYD from the top vs. bottom halves (Fig. $2 \mathrm{H}$; Supplementary Fig. 2C). Individually, AIM2 ${ }^{\mathrm{PYD}}$ and $\mathrm{ASC} \mathrm{CYD}^{\mathrm{P}}$ complexes showed mostly uniform energy landscapes from either filament pole, with both filaments showing more stable interactions at the bottom (Fig. $2 \mathrm{H}$ and Supplementary Fig. 3A, B). The moderate asymmetry suggests that the bottom interfaces might be preferred for homotypic assembly, or it could also reflect the intrinsic noise from sampling multiple conformations in an all-atom MD simulation. Nevertheless, consistent with Rosetta simulations, significantly more energy was required to dissociate $\mathrm{ASC}^{\mathrm{PYD}}$ from the top of the AIM2 ${ }^{\mathrm{PYD}}$ filament than the bottom (Fig. $2 \mathrm{H}$ and Supplementary Fig. 3B). Together, our in silico analyses consistently suggest that individual filaments assemble bidirectionally, AIM2 $2^{\mathrm{PYD}}$ strongly prefers to assemble homotypically, and the recognition between $\mathrm{AIM} 2^{\mathrm{PYD}}$ and $\mathrm{ASC}{ }^{\mathrm{PYD}}$ occurs via the type 2 interface.

In vitro experiments corroborate in silico predictions. To test our simulation results, we first tracked the assembly of fluorlabeled recombinant $\mathrm{AIM} 2^{\mathrm{PYD}}$ and $\mathrm{ASC}{ }^{\mathrm{PYD}}$ filaments via confocal fluorescence microscopy. When we mixed two populations of differentially labeled maltose-binding-protein-tagged (MBP)$\mathrm{AIM} 2^{\mathrm{PYD}}$ at 1:1 ratio and triggered polymerization by cleaving MBP via Tobacco Etch Virus protease $(\mathrm{TEVp})^{19}$, the two colors colocalized in the same filaments (Fig. 3A, AIM2 $2^{\text {PYD }}$ AIM2 ${ }^{\mathrm{PYD}}$ ); differentially labeled ASC ${ }^{\mathrm{PYD}}$ populations also colocalized in the same filaments (Fig. 3A, ASC ${ }^{\mathrm{PYD}} \cdot \mathrm{ASC}^{\mathrm{PYD}}$ ). Importantly, when we preassembled the AIM2PYD filament labeled with one color and added AIM2 $2^{\mathrm{PYD}}$ monomers labeled with a different color, nascent filaments extended from both axial poles of existing filaments (Fig. 3A, (AIM2 ${ }^{\mathrm{PYD}}$ filament) $\left.+\mathrm{AIM} 2^{\mathrm{PYD}}\right) ; \mathrm{ASC}^{\mathrm{PYD}}$ filaments also displayed random bidirectional assembly (Fig. 3A, (ASC ${ }^{\mathrm{PYD}}$ filament) $+\mathrm{ASC}^{\mathrm{PYD}}$ ). These results corroborate that homotypic filaments assemble bidirectionally. Next, we mixed differentially labeled MBP-AIM2 2 PYD and MBP-ASC ${ }^{\mathrm{PYD}}$ at $1: 1$ ratio, and monitored their filament assembly upon triggering polymerization via TEVp. Here, each protein appeared to be oligomerized separately without colocalizing on the same filament, and two distinct filaments interacted only at one specific axial pole (Fig. 3B, $\left.\mathrm{AIM} 2^{\mathrm{PYD}} \cdot \mathrm{ASC}^{\mathrm{PYD}}\right)$. Such a unidirectional interaction was even more evident when we added excess nascent proteins over preformed filaments (Fig. 3B, (AIM2PYD filament) $+\mathrm{ASC}^{\mathrm{PYD}}$ and (ASC ${ }^{\text {PYD }}$ filament) + AIM2 ${ }^{\text {PYD }}$ ). In addition, no significant Förster resonance energy transfer (FRET) signals were observed when we triggered the assembly of a donor-labeled AIM2 $2^{\mathrm{PYD}}$ and acceptor-labeled ASC ${ }^{\mathrm{PYD}}$ (Fig. 3C. see also ref. ${ }^{19}$ ), indicating that $\mathrm{AIM} 2^{\mathrm{PYD}}$ and $\mathrm{ASC}{ }^{\mathrm{PYD}}$ do not co-assemble, yet the recognition entails at least one to be oligomeric. Overall, our biochemical experiments agree with the computational predictions.

Simulations to identify key interfaces that govern the recognition and distinction between AIM2 ${ }^{\text {PYD }}$ and ASC ${ }^{\text {PYD }}$. Our observations thus far suggest that there exist distinct interactions that underpin individual assemblies and those that mediate the recognition between $\mathrm{AIM} 2^{\mathrm{PYD}}$ and $\mathrm{ASC}{ }^{\mathrm{PYD}}$, such as the type 2 interface for the heterotypic recognition. The side chains at the filament interfaces are poorly conserved between AIM2 ${ }^{\text {PYD }}$ and $\mathrm{ASC}^{\mathrm{PYD}}$ (Supplementary Fig. 4A), indicating that diverse 


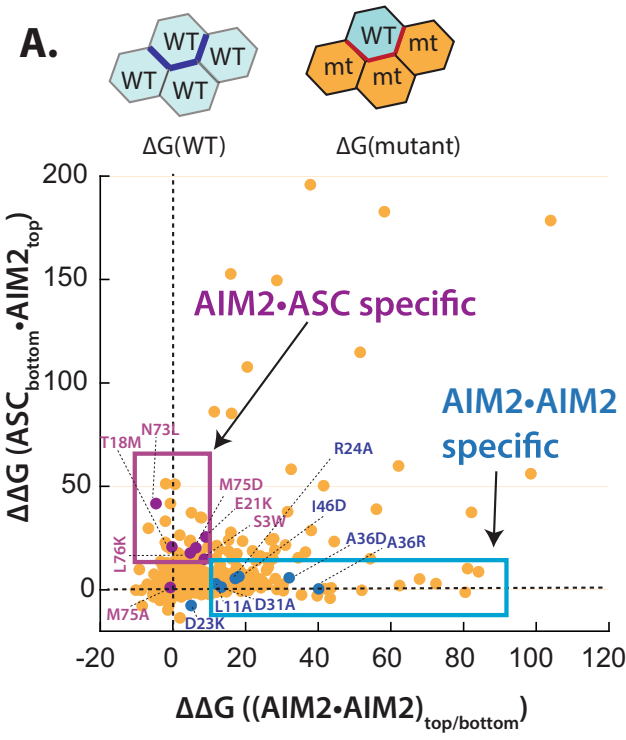

B.

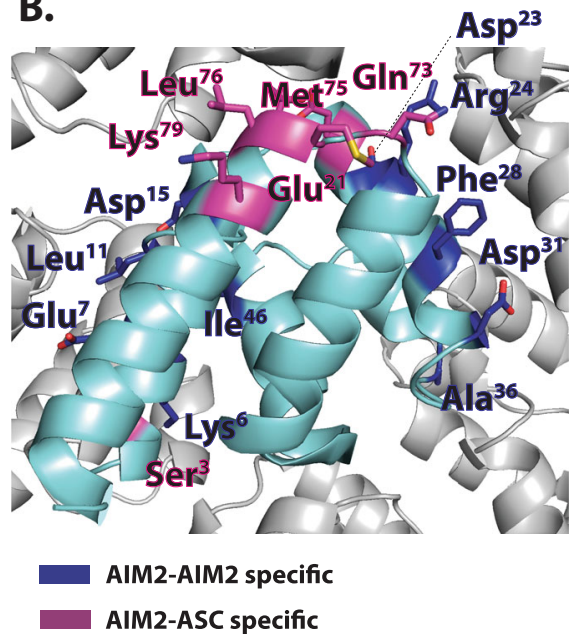

Fig. 4 in silico mutagenesis for identifying side chains that dictate the specificity of the AIM2-ASC inflammasome assembly. A Top: cartoons describing in silico mutagenesis strategy. The ligand monomer was kept WT and the interface residues of pocket monomers were mutated. The $\Delta \Delta \mathrm{G}$ value for each mutated residue was obtained by $\Delta \mathrm{G}_{\text {(WT_ligand•WT_pocket) }}-\Delta \mathrm{G}_{\text {(WT_ligand•mt_pocket) }}$. One pocket is shown as an example, and we applied the same strategy to all six pockets described in Fig. 2 (mt: mutant). Bottom: a plot of AIM2 $2^{\text {PYD }}$ mutations that would interfere with $\mathrm{AIM} 2^{\mathrm{PYD}} \bullet \mathrm{AIM} 2^{\mathrm{PYD}}$ or $\mathrm{AIM} 2^{\mathrm{PYD}} \bullet \mathrm{ASC}^{\mathrm{PYD}}$ interaction. Selected mutations for follow-up biochemical and cellular studies are indicated. B A cartoon of the AIM2 ${ }^{\text {PYD }}$ filament indicating the residues that Rosetta predicts to interfere with either homotypic assembly or ASC PYD recognition when mutated.

side-chain interactions can support the assembly of homologous supra-structures (i.e., redundancy in assembly code). Thus, we decided to forego an alanine-scanning-like approach for identifying and validating key interfaces. Instead, we used Rosetta to suggest mutations that would selectively disrupt homotypic or heterotypic interactions without precluding filament assembly.

Here, we in silico mutated each side chain of pocket protomers that interfaces ligand PYDs to all other a.a. using PyRosetta ${ }^{42}$ (e.g., Fig. 4A top). We then obtained interface energy scores $(\Delta \mathrm{Gs})$ for WT_ligand•WT_pocket and WT_ligand•mt_pocket complexes (Fig. 4A top. WT: wild type, mt: mutant); subtracting the $\Delta \mathrm{G}$ of each WT•mt pair from that of the WT.WT pair then provided the effect of a given mutation $(\Delta \Delta \mathrm{G})$. We then plotted
$\Delta \Delta \mathrm{Gs}$ for $\mathrm{AIM} 2^{\mathrm{PYD}} \cdot \mathrm{AIM}^{\mathrm{PYD}}$ (both top and bottom docking; Fig. $4 \mathrm{~A}$ ) vs. $\Delta \Delta$ Gs for AIM2•ASC complexes (ASC ${ }^{\mathrm{PYD}}$ docking

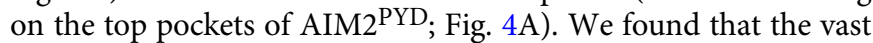
majority of mutations are deleterious for both $\mathrm{AIM} 2^{\mathrm{PYD}}$ - $\mathrm{AIM}_{2}{ }^{\mathrm{PYD}}$ and $\mathrm{AIM} 2^{\mathrm{PYD}} \cdot \mathrm{ASC}^{\mathrm{PYD}}$ interactions (the upper right quadrant in Fig. 4A), suggesting that the a.a. selection has already been optimized for the self-assembly and recognition. Nonetheless, we identified 88 mutations at nine unique side chains, resulting in $\Delta \Delta \mathrm{G}_{\left(\mathrm{AIM} 2 \cdot \mathrm{AIM}_{2}\right)}>10$ and $\Delta \Delta \mathrm{G}_{\left(\mathrm{AIM}_{2} \cdot \mathrm{ASC}^{\mathrm{AYD}}\right)}<10$ (i.e., mutations that would selectively disrupt $\mathrm{AIM} 2^{\mathrm{PYD}} \cdot \mathrm{AIM} 2^{\mathrm{PYD}}$ interactions without abolishing $\mathrm{AIM} 2^{\mathrm{PYD}} \cdot \mathrm{ASC}^{\mathrm{PYD}}$ interactions; boxed area in Fig. 4B and listed in Supplementary Fig. 4B). Interestingly, all these side chains were found on the lateral type 1 and type 3 interfaces, but none at the axial type 2 interfaces (Fig. 4B and Supplementary Fig. 4B). Next, to identify mutations that would selectively disrupt AIM2 ${ }^{\mathrm{PYD}} \cdot \mathrm{ASC}^{\mathrm{PYD}}$ interactions, we looked for those resulted in $\Delta \Delta \mathrm{G}_{\left(\mathrm{AIM} 2 \cdot \mathrm{AIM}_{2}\right)}$ to be $<10$ and $\Delta \Delta \mathrm{G}_{\text {(AIM2.ASC) }}$ to be $>10$. Here, we identified 49 mutations at ten unique AIM2 ${ }^{\mathrm{PYD}}$ side-chain positions, all but one located on the type $2 \mathrm{~b}$ surface (Fig. 4B and Supplementary Fig. 4B). These results are consistent with the mechanism, in which the lateral interfaces drive the assembly of the AIM2 ${ }^{\text {PYD }}$ filament without biasing any directions, while the recognition between $\mathrm{AIM}^{\mathrm{PYD}}$ and $\mathrm{ASC}^{\mathrm{PYD}}$ occurs at the type 2 interface.

In vitro and in cellulo experiments corroborate in silico predictions. Mutations that abolish the self-assembly of $\mathrm{AIM} 2{ }^{\mathrm{PYD}}$ decreases the dsDNA-binding activity of $\mathrm{AIM}_{2} \mathrm{FL}$, as oligomerization is couple to signal recognition ${ }^{17}$. Nevertheless, most of such $\mathrm{AIM} 2^{\mathrm{FL}}$ mutants still assemble into filaments on the dsDNA scaffold ${ }^{17}$. Also of note, AIM2 filaments can self-perpetuate its assembly by accelerating the polymerization of nascent monomers ${ }^{19}$. Thus, to test our simulation results, we generated Rosetta predicted mutations on $\mathrm{AIM}_{2}{ }^{\mathrm{FL}}$ and first confirmed filament formation on dsDNA (Supplementary Fig. 5; all mutants formed filaments except N73L). We then determined whether dsDNA-bound $\mathrm{AIM} 2^{\mathrm{FL}}$ mutants could accelerate the polymerization of FRET donor/acceptor-labeled $\mathrm{AIM}_{2} \mathrm{PYD}$ or $\mathrm{ASC}^{\mathrm{PYD}}$ (Fig. 5A-C and Supplementary Fig. 6; see also ref. ${ }^{19}$ )

As predicted from our simulation, L11A, A36D/R, and I46D were significantly more defective in accelerating the polymerization of $\mathrm{AIM} 2^{\mathrm{PYD}}$ than that of $\mathrm{ASC} \mathrm{PYD}^{\mathrm{PD}}$ (Fig. $5 \mathrm{~A}$ and Supplementary Fig. 6) We also tested D23K as it appeared to enhance the interaction with ASC, while disrupting AIM2-AIM2 interactions (Fig. 4A). We found that D23K-AIM $2^{\mathrm{FL}}$ did not enhance the interaction with $\mathrm{ASC}^{\mathrm{PYD}}$, but was more defective in inducing the polymerization of $\mathrm{AIM}_{2} \mathrm{PYD}$ (Fig. 5A and Supplementary Fig. 6). These results consistently suggest that the lateral surface residues of AIM2 ${ }^{\text {PYD }}$ preferentially, but not exclusively, promote homotypic assembly. Next, again consistent with Rosetta predictions, E21K and M75D were significantly more defective in inducing the polymerization of $\mathrm{ASC}^{\mathrm{PYD}}$ than that of $\mathrm{AIM} 2^{\mathrm{PYD}}$ (Fig. $5 \mathrm{~B}$ and Supplementary Fig. 6), corroborating that the type $2 \mathrm{~b}$ surface of the $\mathrm{AIM} 2^{\mathrm{PYD}}$ filament recruits $\mathrm{ASC} \mathrm{CYD}^{\mathrm{PY}}$ (N73L-AIM2 ${ }^{\mathrm{FL}}$ failed to induce any filament formation consistent with the lack of selfassembly (Fig. 5, and Supplementary Figs. 5 and 6)). Notably, M75A-AIM $2^{\mathrm{FL}}$ (null in Rosetta mutagenesis) retained the WT-like activity (Supplementary Fig. 6), supporting the idea that a simple alanine-scanning approach is inadequate due to the redundancy in assembly code.

We then used the above Rosetta-based approach to identify mutations at the type 2 a surface of ASC ${ }^{\mathrm{PYD}}$ that would selectively disrupt the interaction with AIM2 ${ }^{\mathrm{PYD}}$ (Supplementary Fig. 7A-C). Previously, we found that the ASC ${ }^{\text {PYD }}$ filament accelerates the assembly of AIM2 ${ }^{\text {PYD }}$ via a positive feedback loop ${ }^{19}$. Thus, we 

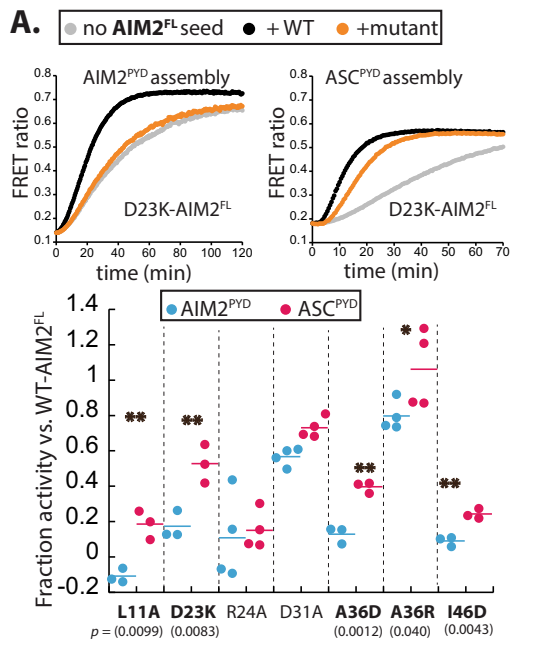

D.
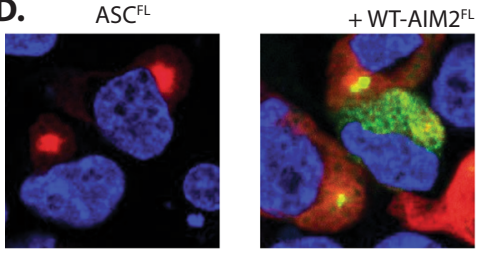

$+\mathrm{A} 36 \mathrm{D}-\mathrm{AIM} 2^{\mathrm{FL}}$
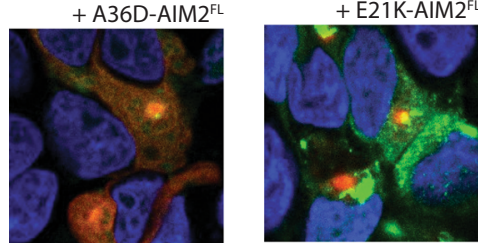
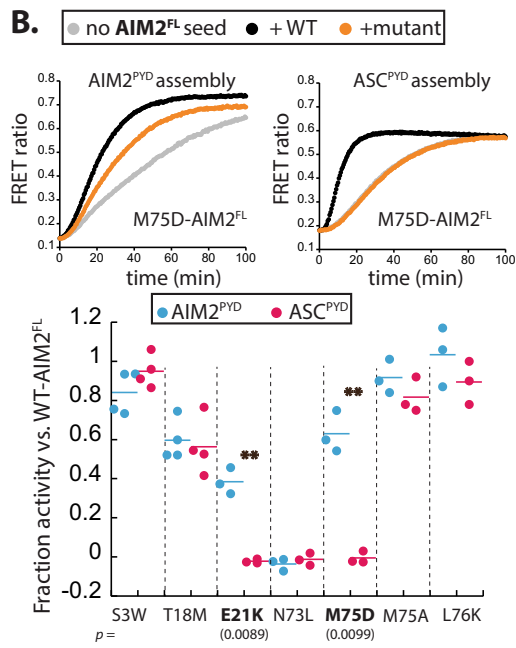

+ L11A-AIM2 $2^{\mathrm{FL}}$
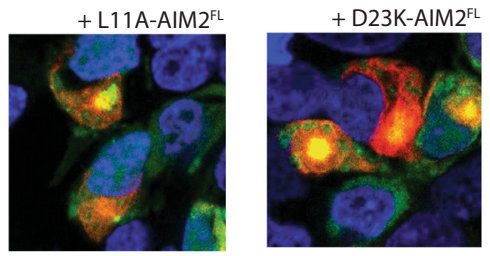

$+\mathrm{M} 75 \mathrm{D}-\mathrm{AIM} 2^{\mathrm{FL}}$
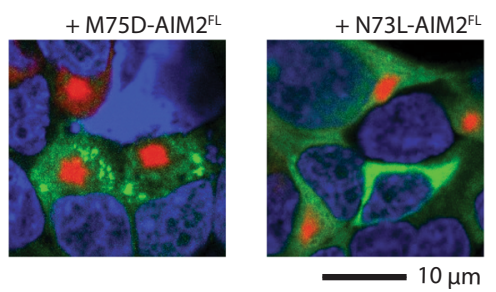
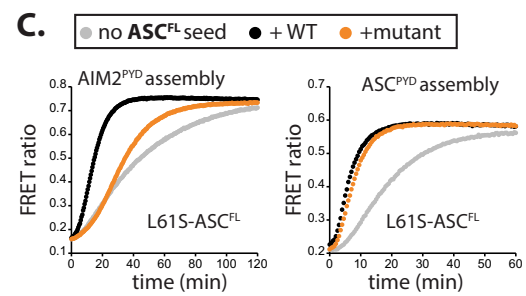

- $\mathrm{AIM2}^{\mathrm{PYD}} \cdot \mathrm{ASC}^{\mathrm{PYD}}$

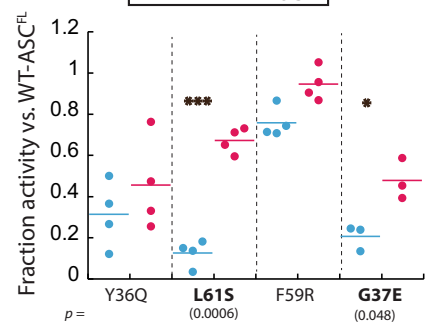

E.

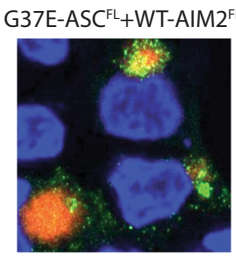

L61S-ASC ${ }^{\mathrm{FL}}+$ WT-AIM2 ${ }^{\mathrm{FL}}$

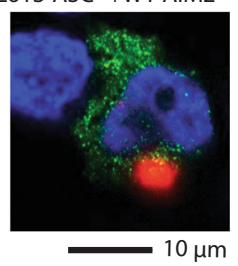

Fig. 5 biochemical and cellular experiments support Rosetta predictions. A Testing AIM2 $2^{\mathrm{FL}}$ mutants predicted to interfere with homotypic assembly. B Testing AIM2 ${ }^{\mathrm{FL}}$ mutants predicted to interfere ASC ${ }^{\mathrm{PYD}}$ recognition. C Testing ASC $\mathrm{FL}^{\mathrm{FL}}$ mutants predicted to interfere with $\mathrm{AIM} 2^{\mathrm{PYD}}$ recognition. For $\mathbf{A}-\mathbf{C}$, sample plots show the time-dependent polymerization of FRET donor- and acceptor-labeled AIM2 ${ }^{\text {PYD }}$ or ASCPYD in the presence or absence of dsDNAbound (linear plasmid $~ 5$-kilo base-pairs (kbps)) WT or mutant AIM2 FL. Dot plots summarizing the effect of Rosetta predicted AIM2 mutants that would selectively interfere with its own assembly. Polymerization half-times were normalized using WT AIM2 ${ }^{\mathrm{FL}}$-induced assembly (set to 1) and labeled PYDs alone (set to 0 ). ${ }^{\star} p<0.05 ;{ }^{\star \star} p<0.01 ;{ }^{\star \star \star} p<0.001$. $P$ values were calculated using Student's $t$ test for paired samples. D Confocal microscope images of HEK293T cells (co)-transfected with WT ASCFL-mCherry alone. WT ASCFL-mCherry plus WT or mutant AIM2FL-eGFP. E Confocal microscope images of HEK293T cells (co)-transfected with mutant ASC FL-mCherry plus WT AIM2 ${ }^{\mathrm{FL}}$-eGFP. The nucleus is stained with DAPI in both $\mathbf{D}$ and $\mathbf{E}$. Images shown in $\mathbf{D}$ and $\mathbf{E}$ are representative of at least three independent experiments.

generated Rosetta predicted mutations on full-length ASC $\left(\mathrm{ASC}^{\mathrm{FL}}\right.$ ) and tested their capacity for inducing the polymerization of FRET-labeled $\mathrm{ASC}^{\mathrm{PYD}}$ or $\mathrm{AIM} 2^{\mathrm{PYD}}$. We used $\mathrm{ASC}^{\mathrm{FL}}$, as the Cterminal CARD would promote the polymerization of ASC ${ }^{\mathrm{PYD}}$ even if mutations were too deleterious. We found that L61S and G37E were significantly more defective in accelerating the assembly of $\mathrm{AIM}_{2}{ }^{\mathrm{PYD}}$ than that of $\mathrm{ASC} \mathrm{CYD}^{\mathrm{PYD}}$, corroborating that the type 2a surface of $\mathrm{ASC}^{\mathrm{PYD}}$ recognizes $\mathrm{AIM}^{\mathrm{PYD}}$ (Fig. $5 \mathrm{C}$ and Supplementary Fig. 7D).

We next probe the interactions among $\mathrm{AIM}_{2} \mathrm{FL}$ and $\mathrm{ASC}^{\mathrm{FL}} \mathrm{WT}$ and mutants in HEK293T cells. WT AIM2 ${ }^{\mathrm{FL}}$ (ASC ${ }^{\mathrm{FL}}$ ) tagged with $\mathrm{C}$-terminal eGFP or mCherry colocalized in the same complexes as expected (Supplementary Fig. 8). AIM2 ${ }^{\mathrm{FL}}$ showed filamentous complexes that often tangled up into speck-like clusters, while ASC $^{\mathrm{FL}}$ displayed large puncta, as previously reported ${ }^{43}$ (Fig. 5D left, Supplementary Fig. 8A-C). AIM $2 \mathrm{FL}_{-\mathrm{eGFP}}$ mutants coloca-

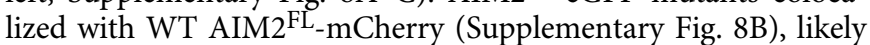
due to assembling/binding on the same (transfected) dsDNA strands as WT. Interestingly, when $\mathrm{AIM}_{2} \mathrm{FL}_{-} \mathrm{eGFP}$ and $\mathrm{ASC}^{\mathrm{FL}}$ mCherry were co-transfected, $\mathrm{ASC}^{\mathrm{FL}}$ filaments further expanded as if $\mathrm{ASC}^{\mathrm{FL}}$ assembles from multiple $\mathrm{AIM} 2^{\mathrm{FL}}$ foci (Fig. 5D, (+WT $\left.\mathrm{AIM} 2^{\mathrm{FL}}\right)$ ). When we co-transfected $\mathrm{AIM} 2^{\mathrm{FL}}$-eGFP mutants and $\mathrm{WT} \mathrm{ASC}^{\mathrm{FL}}$-mCherry, those that preferentially decreased the ability to interact with AIM2 ${ }^{\mathrm{PYD}}$ still resulted in expanded $\mathrm{ASC} \mathrm{C}^{\mathrm{FL}}$ complexes as observed from WT (Fig. 5D, L11A, D23K, and $\mathrm{A} 36 \mathrm{D})$. By contrast, $\mathrm{ASC}^{\mathrm{FL}}$ stayed as a single punctum when cotransfected with $\mathrm{AIM} 2^{\mathrm{FL}}$ mutants that failed to accelerate the polymerization of $\mathrm{ASC}^{\mathrm{PYD}}$ (E21K and M75D; Fig. 5D, E21K and M75D); N73L-AIM2 ${ }^{\mathrm{FL}}$-eGFP, which cannot oligomerize (Supplementary Figs. 5 and 8A), also failed to interact with ASC ${ }^{\mathrm{FL}}$-mCherry (Fig. 5D, N73L). We also imaged G37E- and L61S-ASC ${ }^{\mathrm{FL}}$-mCherry with eGFP-tagged WT $\mathrm{AIM} 2^{\mathrm{FL}}$ and $\mathrm{ASC}^{\mathrm{FL}}$. The $\mathrm{ASC}^{\mathrm{FL}}$ mutants still showed large puncta and also colocalized with WT (Supplementary Fig. 8C). However, WT $\mathrm{AIM}_{2} \mathrm{FL}_{-} \mathrm{eGFP}$ failed to colocalize or induce the expansion of these mutants when co-transfected (Fig. 5E). Together, our in vitro and in cellulo experiments consistently support our in silico predictions, in which unique lateral and axial interfaces within homologous filaments dictate their recognition and distinction. 
A.
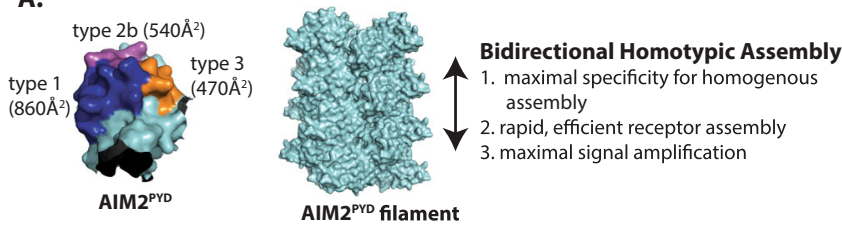

B.

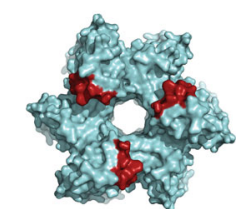

type 1

$\left(2,450 \AA^{2}\right)$

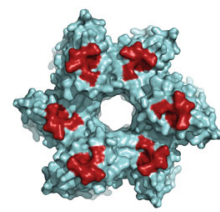

type $\mathbf{2 b}$

$\left(2,670 \AA^{2}\right)$

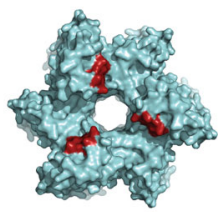

type 3

$\left(1,060 \AA^{2}\right)$

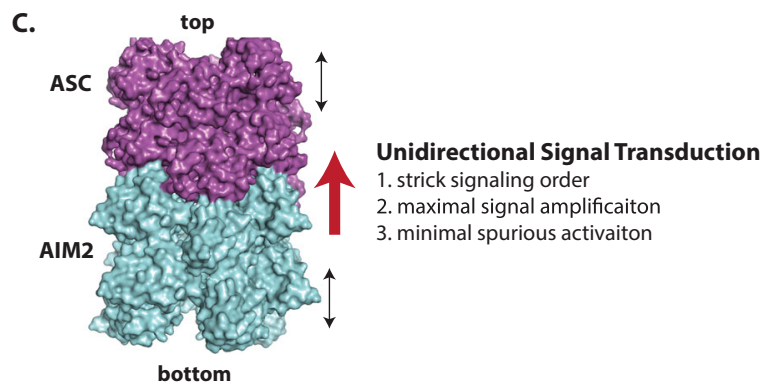

Fig. 6 Strategies for signal transduction by the AIM2-ASC inflammasome. A Left: a surface representation of AIM2 ${ }^{\text {PYD }}$ monomer. The buried surface area in the filament for each interface type is indicated. Right: a scheme describing the advantages of bidirectional homotypic assembly. B The top surface view of the AIM2PYD filament. Each solvent accessible interface is colored in red with the calculated surface area. C A scheme describing the advantages of unidirectional signal transduction by the AIM2-ASC inflammasome.

\section{Discussion}

Inflammasomes transduce signals by assembling supramolecular structures $^{14,17-20,22,23}$. It is well accepted that architectural complementarity is important for the recognition between primary signaling components $21-23,28,30,34$. Indeed, our AIM2 ${ }^{\text {PYD }}$ filament structure further cements this concept. Nevertheless, these observations further highlight a long-standing question as to how such homologous filaments distinguish and recognize one another. Here, we set forth a broadly applicable research platform for answering these questions and propose design principles that define the signaling mechanisms of inflammasomes.

Strategies for homotypic filament assembly. Our experiments consistently show that the assembly of individual filaments occur bidirectionally, with lateral type 1 and type 3 interfaces (especially type 1) of $\mathrm{AIM} 2{ }^{\mathrm{PYD}}$ favoring homotypic interactions, while still supporting the recognition of $\mathrm{ASC} \mathrm{PYD}^{\mathrm{P}}$. The lateral type 1 surfaces are the largest in any PYDs (Fig. 6A left), which would be ideal for recognizing other homotypic protomers to initiate assembly without any prescribed directionalities. The lack of directionality in homotypic assembly would then allow AIM2 to maximally benefit from one-dimensional random diffusion on pathogenic $\mathrm{dsDNA}^{19}$, resulting in a timely response by the upstream receptor (Fig. 6A right). Interestingly, the bidirectional assembly is in contrast to other cytoskeletal and signaling filaments such as actin $^{44}$ and B-cell lymphoma 10 (BCL10) ${ }^{39}$. Considering that both actin and BCL10 filaments originate from cell membranes/ defined borders ${ }^{39,44}$, it is tempting to speculate that the bidirectional assembly of inflammasome filaments have evolved to take full advantage of no immediate boundaries in the cytosol.
Strategies for signaling by assembly. The AIM2 ${ }^{\mathrm{PYD}}$ filament displays higher stability than either the ASC ${ }^{\mathrm{PYD}}$ filament or AIM2PYD.ASC ${ }^{\text {PYD }}$ complex (Fig. 2), which would ensure homotypic assembly of the receptor filament especially within the dsDNA scaffold. On the other hand, the interaction between $\mathrm{AIM}_{2} \mathrm{PYD}$ and $\mathrm{ASC} \mathrm{CYD}^{\mathrm{PY}}$ is more favorable than homotypic ASCPYD interactions at a specific axial pole (Fig. 2). In addition, $\mathrm{AIM} 2^{\mathrm{PYD}}$ and $\mathrm{ASC}{ }^{\mathrm{PYD}}$ recognize each other only when at least one is oligomeric (Fig. 3B). Of note, electrostatic surface analyses suggest that the charge complementary is reversed at the type 1 interface for AIM2 and ASC, likely indicating that the heterologous interactions between the monomers are not favorable (i.e., the type 1a surface of AIM2 ${ }^{\mathrm{PYD}}$ is largely basic, whereases that of ASC PYD is acidic; Supplementary Fig. 9A). Importantly, our in silico, in vitro, and in cellulo experiments consistently demonstrate that the directional interaction at the type 2 interface is most critical (Figs. 2-5). The surface area of the type 2 interface is much smaller than that of the type 1 interface in monomeric PYDs (Fig. 6A). However, because of the axial location, the type $2 \mathrm{~b}$ surfaces become as accessible as the type 1 surfaces once $\mathrm{AIM} 2^{\mathrm{PYD}}$ assembles into a filament (Fig. 6B). Moreover, electrostatic surface analyses suggest that the bottom of the AIM2 ${ }^{\mathrm{PYD}}$ filament is unfavorable for interacting with the ASC ${ }^{\mathrm{PYD}}$ filament due to highly positively charged surfaces (Supplementary Fig. 9B). We propose that such conditional scaffolding by the upstream filament not only ensures proper signal transduction orders, but also maximizes signal amplification (Fig. 6C). For instance, inflammasomes assemble in a digital fashion and entail cell death $^{14,19}$. Thus, it must be imperative that ASC does not polymerize unless upstream receptors are fully activated by correct signals. Thus, the sequential/conditional assembly of the AIM2ASC inflammasome would minimize its spurious activity in the absence of bona fide danger signals. Of note, not only the assembly, but also the signaling activity of AIM2 depends on dsDNA length, which regulates the probability of assembling the intact axial base of its filament ${ }^{19}$. Our results here further explain the dsDNA length-dependent mechanism, as the intact filament base would conditionally maximize the presence of the type $2 \mathrm{~b}$ surfaces to recruit ASC (Fig. 6B). Subsequently, such a stringent recognition mechanism would then ensure that ASC polymerizes homogenously via its prion-like assembly mechanism ${ }^{45}$, resulting in maximal signal amplification (Fig. 6C). Alternatively, if AIM2 and ASC were to either co-assemble or interact without distinct energetic hierarchies, we envision that upstream and downstream oligomers would either alternate or even cap their respective assemblies, resulting in signal attenuation.

Future directions. Our successful implementation of Rosetta to decode the specificity of the AIM2-ASC inflammasome suggests that our approach can be broadly applied to other homologous signaling filaments. However, we noted that Rosetta was correct at $\sim 50 \%$ in predicting energetically important mutations (Fig. 5), indicating that there is room for improvement. Nonetheless, given that precisely pinpointing the role of individual residues is intrinsically challenging, we find the Rosetta suite to be an excellent tool for decoding the specificity of the filamentous assemblies.

It was recently postulated that the directionality of the NLRP6 ${ }^{\mathrm{PYD}}$-ASC ${ }^{\mathrm{PYD}}$ interaction would be the same as what we found here for $\mathrm{AIM}^{\mathrm{PYD}}-\mathrm{ASC}^{\mathrm{PYD}}$ (ref. ${ }^{20}$ ). Thus, it is tempting to speculate that recruiting ASC via its type $2 \mathrm{a}$ surface by the type $2 \mathrm{~b}$ surface of upstream receptors is the universal strategy in inflammasome signaling. Other than NLRP6 and AIM2, there are at least 14 other upstream receptors that signals through ASC ${ }^{1,14}$. Future investigations will reveal to what extent the mechanisms we 
found for AIM2 apply in other receptors and how well Rosetta fares in answering these questions.

\section{Methods}

Protein expression and purification. Human $\mathrm{AIM} 2^{\mathrm{FL}}$ (residues 1-343), $\mathrm{AIM} 2^{\mathrm{PYD}}$ (residues 1-94) S94C for fluorophore labeling, $\mathrm{ASC}^{\mathrm{PYD}}$ (residues 1-92) were cloned into the pET28b vector (Novagen) with an N-terminal MBP tag and TEVp recognition site. For cryo-EM, we and found a construct including $\sim 20$ a.a. in the unstructured linker region (residues 1-117) resulted in well-separated filaments (denoted as AIM2 ${ }^{\mathrm{IRND}}$; Fig. $1 \mathrm{~A}$ ). ASC ${ }^{\mathrm{FL}}$ was cloned with the MBP tag at both Nand C-termini with the TEVp recognition site flanking $\mathrm{MBP}$ and $\mathrm{ASC}{ }^{\mathrm{FL}}$. All proteins were expressed in Escherichia coli BL21 Rosetta $2^{\mathrm{DE} 3}$ and purified using affinity (MBP/amylose), cation exchange, and followed by size-exclusion chromatography. Proteins were then concentrated and stored at $-80^{\circ} \mathrm{C}$, see also refs. ${ }^{17,19}$ (all primers generated for this study are listed in Supplementary Table 2).

Cryo-EM sample preparation. A total of $5 \mu \mathrm{l}$ sample of $2.75 \mu \mathrm{M}$ AIM2 $2^{\text {IRND }}$ (cleaved for 30 min with $6 \mu \mathrm{M}$ TEVp) was applied to Lacey grids, followed by automatically blotting for $1.5 \mathrm{~s}$ and plunge freezing, using the FEI Vitrobot Mark IV operated at $100 \%$ humidity and room temperature (Johns Hopkins University). Cryo-EM data were collected at the National Cancer Institute National cryo-EM facility (NCI NCEF, Frederick, MD) using the FEI Titan equipped with the Gatan $\mathrm{K} 2$ direct electron detector operating at $300 \mathrm{keV}$, using the super-resolution mode $(0.66 \AA$ /pixel). A total of 2700 micrographs were collected from one grid at a defocus range from -1.0 to $-2.5 \mu \mathrm{m}$. Each micrograph was equally fractioned into 40 frames with a total exposure time of $12 \mathrm{~s}$ and a total dose of 42 electrons/ $\AA^{2}$. Data collection statistics are listed in Supplementary Table 1.

Helical reconstruction and model building. The 2700 micrographs were binned by two (to $1.32 \AA / \mathrm{px}$ ) and frames were aligned using MotionCor2 (ref. ${ }^{46}$ ). The defocus values and astigmatism of the micrographs were determined by CTFFIND3 for the aligned full-dose micrographs ${ }^{47}$. A total of 976 micrographs were selected (images with good CTF determination and defocus $<3 \mu \mathrm{m}$ ) for subsequent image processing. CTF was corrected by multiplying the micrographs (only first 20 frames were aligned with a total dose of $\sim 20$ electrons $/ \AA^{2}$ ) with the theoretical CTF, which both corrects the phases and improves the signal-to-noise ratio. The e2helixboxer program in EMAN2 software package ${ }^{48}$ was used for boxing long filaments from the full-dose images. The CTF-corrected micrographs were used for the segment extraction, with a total of 116,285 384 px-long overlapping segments (with a shift of 1.5 times of axial rise) generated. The SPIDER software package ${ }^{49}$ was used for subsequent processing and reconstruction. Using a featureless cylinder as an initial reference, 99,237 segments were used in IHRSR program for the final reconstruction after the helical parameters (an azimuthal rotation of $53.3^{\circ}$ and an axial rise of $14 \AA$ per subunit) converged. The resolution of the final reconstruction was estimated by the FSC between two independent half maps, which shows $3.2 \AA$ at FSC $=0.143$.

We used the crystal structure of AIM2PYD (PDB ID: 4O7Q) as an initial template to dock into the AIM2 ${ }^{\mathrm{PYD}}$ cryo-EM map by rigid body fitting, and then manually edited the model in Chimera ${ }^{50}$ and Coot $^{51}$. We then used the modified model as the starting template to further refine against the segmented cryo-EM map using RosettaCM ${ }^{52}$. The refined monomeric model of AIM2 $2^{\text {PYD }}$ was then rebuilt by RosettaCM with helical symmetry and real-space refined, using Phenix ${ }^{53}$ to improve the stereochemistry, as well as the model-map correlation coefficient. The AIM2 ${ }^{\text {PYD }}$ filament model was validated with MolProbity ${ }^{54}$. Refinement statistics are listed in Supplementary Table 1.

Rosetta docking. The Rosetta Local Docking protocol ${ }^{40,55}$ was applied to Rosetta symmetry relaxed structures of the AIM2 $2^{\mathrm{PYD}}$ cryo-EM structure and ASC $\mathrm{PYD}^{\text {cryo- }}$ EM structure (PDB ID: 3J63). Each complex contained a pocket consisting of three PYDs, and one ligand PYD. The initial position of the ligand was already in the pocket to minimize the search space, as suggested by the local docking protocol. The docking simulation was done 5000 times for each fragment, and the results were analyzed by looking at the interface energy and RMSD from the initial position.

Interface energy analysis and in silico mutagenesis. We used the InterfaceAnalyzer script in Rosetta at individual interfaces of the honeycomb to obtain interaction energies.

Using PyRosetta, we first in silico mutated each interface residue of all $\mathrm{AIM} 2^{\mathrm{PYD}} / \mathrm{ASC} \mathrm{CYD}^{\mathrm{PYD}}$ protomers in the honeycomb into all other possible a.a. ${ }^{42}$. We then removed those that resulted in energy scores $>2$ standard deviations from the mean (e.g., prolines that would distort backbone geometry and cause potential folding issues). We then applied remaining mutants ( 942 possibilities) to the pocket protomers of all six subsections, leaving the ligand protomers as WT. We then used the dG_separate subroutine in Rosetta InterfaceAnalyzer to obtain $\Delta \mathrm{Gs}$ for (WT_ligand•WT_pocket) and (WT_ligand•mutant_pocket) interfaces subtracting the $\Delta \mathrm{G}$ of each mutant from that of WT then provides the changes in interface energy caused by the mutation $(\Delta \Delta \mathrm{G})$. Each mutant was tested at least three times and the average values were used for analyses.

Molecular dynamics. The coordinates for each protein-ligand complex were obtained from Rosetta docking experiments such that the interface energy score and interface RMSD were minimized. All MD simulations were performed using GROMACS 5.1.3 (gromacs.org) with an all-atom CHARMM36 (ref. ${ }^{56}$ ) force field Simulation scripts were created using CHARMM-GUI ${ }^{57,58}$. Initial coordinates were energy minimized using the steepest descent algorithm and subsequently equilibrated for $4.75 \mathrm{~ns}$, starting in the NVT ensemble and transitioning to the NPT ensemble. Neutralizing ions were added with $\sim 200 \mathrm{Cl}$ and $\sim 200 \mathrm{~K}$ to a box of $13 \mathrm{~nm} \times 13 \mathrm{~nm} \times 13 \mathrm{~nm}$.

Following initial energy minimization and equilibration, a second step of equilibration was performed in the NPT ensemble with a $2 \mathrm{fs}$ timestep for $50 \mathrm{~ns}$. A Nose-Hoover ${ }^{59}$ thermostat was used to maintain a reference temperature of $300 \mathrm{~K}$ with a 1 ps coupling time constant. The protein and solvent were coupled to separate temperature baths. A Parrinello-Rahman ${ }^{6}$ isotropic barostat with a 5 ps coupling time constant was used to maintain a pressure of 1 bar. Particle Mesh Ewald (PME) with a $1.2 \mathrm{~nm}$ cutoff radius, a $0.12 \mathrm{~nm}$ Fourier spacing, and cubic interpolation of 4 were used for electrostatics ${ }^{60}$. Van der Waals interactions had a $1.2 \mathrm{~nm}$ cutoff radius. A LINCS algorithm was used for bond constraints and XYZ periodic boundary conditions were enforced ${ }^{61}$.

Following the second step of equilibration, well-tempered Metadynamics (MetaD) simulations were performed using GROMACS 5.1.3 patched with PLUMED2 (ref. ${ }^{62}$ ), using a CHARMM36 force field. The collective variable $(\mathrm{CV})$ was the distance between the center-of-mass of the pocket and the center-of-mass of the ligand (residue 60). The backbone RMSD stayed mostly constant during the course of simulation (Supplementary Fig. 3A). Gaussians of energy were deposited along the trajectory in this CV space. Gaussians had an initial hill height of $1 \mathrm{~kJ} /$ mol and a width of $0.05 \mathrm{~nm}$. Gaussians were deposited every $400 \mathrm{fs}$. A bias factor of 4 was used to adjust the hill heights according to the well-tempered MetaD scheme. Gaussians were saved to a grid with a bin spacing $0.01 \mathrm{~nm}$. Simulations were considered complete when the ligand completely dissociated from the pocket, i.e. the $\mathrm{CV}$ distance exceeded $5 \mathrm{~nm}$. Positional restraints were placed on every alphacarbon in the pocket to prevent dissociation of the pocket protomers during the entirety of the MetaD simulations. The sum of all deposited Gaussians was computed to represent the dissociation free energy.

Polymerization assays. A total of $100 \mathrm{nM}$ of $\mathrm{AIM} 2^{\mathrm{FL}}$ was cleaved by $6 \mu \mathrm{M}$ TEVp for $20 \mathrm{~min}$ in a 384-well plate. After cleavage, $150 \mathrm{nM}$ of linearized plasmid dsDNA ( 5 -kbps, binding site normalized) was added and allowed to bind for $30 \mathrm{~min}$. To start the assay, $2 \mu \mathrm{M}$ FRET mix of MBP-tagged AIM2 ${ }^{\mathrm{PYD}}$ or $\mathrm{ASC}^{\mathrm{PYD}}$ was added to the same well containing TEVp. Each experiment consisted of a control well with no $\mathrm{AIM} 2^{\mathrm{FL}}$, one with AIM2 ${ }^{\mathrm{FL}}$ WT, and multiple AIM2 ${ }^{\mathrm{FL}}$ mutants for both $\mathrm{AIM} 2^{\mathrm{PYD}}$ and ASC ${ }^{\mathrm{PYD}}$ wells. AIM2 and ASC samples were run at the same time to ensure proper statistical analyses. Half-times for polymerization were calculated and converted to apparent kinetic rates ${ }^{19,63}$. The no AIM2 $2^{\mathrm{FL}}$ control and AIM2 $2^{\mathrm{FL}}$ WT control were used to normalize the kinetics rates for each mutant into an activity ratio scaling from 0 (no $\mathrm{AIM} 2^{\mathrm{FL}}$ present) to 1 (AIM2 ${ }^{\mathrm{FL}} \mathrm{WT}$ activity). $P$ values were calculated using Student's $t$ test for paired samples. The same strategy was used for ASC ${ }^{\mathrm{FL}}$ WT and mutants $(0.5 \mu \mathrm{M}$, precleaved by TEVp for $30 \mathrm{~min})$ inducing the polymerization of FRET-labeled AIM2 $2^{\mathrm{PYD}}$ or ASC ${ }^{\mathrm{PYD}}$.

nsEM. AIM2 ${ }^{\mathrm{FL}}$ bound to dsDNA was prepared in the same manner as for the polymerization assays (100 $\mathrm{nM}$ protein, $150 \mathrm{nM}$ dsDNA, linear plasmid $\sim 5 \mathrm{kilo}$ bps). The samples were applied to carbon coated grids and imaged 19,64 .

Imaging recombinant AIM2 ${ }^{\text {PYD }}$ and ASC $^{\text {PYD }}$ filaments. Filament assembly of Alexa $_{488^{-}}$or Dylight ${ }_{55}$-labeled MBP-AIM2 ${ }^{\text {PYD }}$ and MBP-ASC ${ }^{\text {PYD }}(1 \mu \mathrm{M}$ each or $3 \mu \mathrm{M}$ of nascent proteins for Fig. $3 \mathrm{~A}$ ) was induced by removing MBP by TEVp as indicated in figure legends. For preassembly, the AIM2 ${ }^{P Y D}$ or ASC ${ }^{P Y D}$ filament was cleaved and incubated for $30 \mathrm{~min}$ prior to adding nascent proteins. Images were then taken using a Zeiss Axioskop 50 with a Zeiss Axiocam HRC camera and an Axio Observer inverted microscope with LSM700 confocal module.

Imaging AIM2 ${ }^{\mathrm{FL}}$-eGFP/mCherry and ASC ${ }^{\mathrm{FL}}$-eGFP/mCherry in HEK293T cells $\mathrm{AIM} 2{ }^{\mathrm{FL}}$ and $\mathrm{ASC} \mathrm{FL}^{\mathrm{FL}}$ variants were cloned into the $\mathrm{pCMV} 6$ vector harboring eGFP or mCherry. To preserve native PYD-PYD interactions, the fluorescent proteins were positioned at the $\mathrm{C}$-terminus of $\mathrm{AIM} 2^{\mathrm{FL}}$ or $\mathrm{ASC}^{\mathrm{FL}}$. Plasmids were then transiently transfected into HEK293T cells using lipofectamine $(0.5 \mu \mathrm{g}$ each plasmid; Invitrogen). After $12 \mathrm{~h}$, cells were fixed with $4 \%$ paraformaldehyde and mounted on glass slides using ProLong Gold Antifade Mountant with DAPI (Thermo Fisher). Cells were then imaged using the same confocal microscope as the recombinant proteins. 
Reporting summary. Further information on research design is available in the Nature Research Reporting Summary linked to this article.

\section{Data availability}

The cryo-EM structure has been deposited to the Protein Data Bank, PDB ID: 7K3R. The corresponding cryo-EM map was deposited in the EMDB with access code EMD-22656. The datasets generated during and/or analyzed during this study are available from the corresponding author on request. Source data are provided with this paper.

\section{Code availability}

All MD scripts can be found at https://github.com/nav610/a2dissocation ${ }^{65}$.

Received: 17 September 2020; Accepted: 14 April 2021;

Published online: 12 May 2021

\section{References}

1. Broz, P. \& Dixit, V. M. Inflammasomes: mechanism of assembly, regulation and signalling. Nat. Rev. Immunol. 16, 407-420 (2016).

2. Bandera, A. et al. The NLRP3 inflammasome is upregulated in HIV-infected antiretroviral therapy-treated individuals with defective immune recovery. Front. Immunol. 9, 214 (2018).

3. Maruzuru, Y. et al. Herpes simplex virus 1 VP22 inhibits AIM2-dependent inflammasome activation to enable efficient viral replication. Cell Host Microbe 23, 254-265 e7 (2018).

4. Kim, S. et al. Listeria monocytogenes is sensed by the NLRP3 and AIM2 inflammasome. Eur. J. Immunol. 40, 1545-1551 (2010).

5. Reyes Ruiz, V. M. et al. Broad detection of bacterial type III secretion system and flagellin proteins by the human NAIP/NLRC4 inflammasome. Proc. Natl Acad. Sci. USA 114, 13242-13247 (2017).

6. Fernandes-Alnemri, T. et al. The AIM2 inflammasome is critical for innate immunity to Francisella tularensis. Nat. Immunol. 11, 385-393 (2010).

7. Yang, C. A. \& Chiang, B. L. Inflammasomes and human autoimmunity: a comprehensive review. J. Autoimmun. 61, 1-8 (2015).

8. Yi, Y. S. Role of inflammasomes in inflammatory autoimmune rheumatic diseases. Korean J. Physiol. Pharm. 22, 1-15 (2018)

9. Yap, J. K. Y., Moriyama, M. \& Iwasaki, A. Inflammasomes and pyroptosis as therapeutic targets for COVID-19. J. Immunol. 205, 307-312 (2020).

10. Henao-Mejia, J., Elinav, E., Thaiss, C. A. \& Flavell, R. A. Inflammasomes and metabolic disease. Annu. Rev. Physiol. 76, 57-78 (2014).

11. De Nardo, D. \& Latz, E. NLRP3 inflammasomes link inflammation and metabolic disease. Trends Immunol. 32, 373-379 (2011).

12. Man, S. M., Karki, R. \& Kanneganti, T. D. AIM2 inflammasome in infection, cancer, and autoimmunity: role in DNA sensing, inflammation, and innate immunity. Eur. J. Immunol. 46, 269-280 (2016).

13. Lin, C. \& Zhang, J. Inflammasomes in inflammation-induced cancer. Front Immunol. 8, 271 (2017).

14. Lu, A. \& Wu, H. Structural mechanisms of inflammasome assembly. FEBS J. 282, 435-444 (2015)

15. Bami, S. et al. The use of anakinra in the treatment of secondary hemophagocytic lymphohistiocytosis. Pediatr. Blood Cancer 67, e28581 (2020).

16. Fernandes-Alnemri, T., Yu, J. W., Datta, P., Wu, J. \& Alnemri, E. S. AIM2 activates the inflammasome and cell death in response to cytoplasmic DNA. Nature 458, 509-513 (2009).

17. Morrone, S. R. et al. Assembly-driven activation of the AIM2 foreign-dsDNA sensor provides a polymerization template for downstream ASC. Nat. Commun. 6, 7827 (2015).

18. Lu, A. et al. Unified polymerization mechanism for the assembly of ASC dependent inflammasomes. Cell 156, 1193-1206 (2014).

19. Matyszewski, M., Morrone, S. R. \& Sohn, J. Digital signaling network drives the assembly of the AIM2-ASC inflammasome. Proc. Natl Acad. Sci. USA 115, E1963-E1972 (2018)

20. Shen, C. et al. Molecular mechanism for NLRP6 inflammasome assembly and activation. Proc. Natl Acad. Sci. USA 116, 2052-2057 (2019).

21. Li, Y. et al. Cryo-EM structures of ASC and NLRC4 CARD filaments reveal a unified mechanism of nucleation and activation of caspase-1. Proc. Natl Acad. Sci. USA 115, 10845-10852 (2018).

22. Lu, A. et al. Molecular basis of caspase-1 polymerization and its inhibition by a new capping mechanism. Nat. Struct. Mol. Biol. 23, 416-425 (2016).

23. Matyszewski, M. et al. Cryo-EM structure of the NLRC4(CARD) filament provides insights into how symmetric and asymmetric supramolecular structures drive inflammasome assembly. J. Biol. Chem. 293, 20240-20248 (2018).

24. Zhang, L. et al. Cryo-EM structure of the activated NAIP2-NLRC4 inflammasome reveals nucleated polymerization. Science 350, 404-409 (2015).

25. Tenthorey, J. L. et al. The structural basis of flagellin detection by NAIP5: a strategy to limit pathogen immune evasion. Science 358, 888-893 (2017).

26. Kagan, J. C., Magupalli, V. G. \& Wu, H. SMOCs: supramolecular organizing centres that control innate immunity. Nat. Rev. Immunol. 14, 821-826 (2014).

27. Kumari, P., Russo, A.J., Shivcharan, S. \& Rathinam, V.A. AIM2 in health and disease: inflammasome and beyond. Immunol. Rev. 297, 83-95 (2020).

28. Hornung, V. et al. AIM2 recognizes cytosolic dsDNA and forms a caspase-1activating inflammasome with ASC. Nature 458, 514-518 (2009).

29. Wu, J., Fernandes-Alnemri, T. \& Alnemri, E. S. Involvement of the AIM2, NLRC4, and NLRP3 inflammasomes in caspase-1 activation by Listeria monocytogenes. J. Clin. Immunol. 30, 693-702 (2010).

30. Denes, A. et al. AIM2 and NLRC4 inflammasomes contribute with ASC to acute brain injury independently of NLRP3. Proc. Natl Acad. Sci. USA 112, 4050-4055 (2015).

31. Lammert, C. R. et al. AIM2 inflammasome surveillance of DNA damage shapes neurodevelopment. Nature 580, 647-652 (2020).

32. $\mathrm{Hu}, \mathrm{B}$. et al. The DNA-sensing AIM2 inflammasome controls radiationinduced cell death and tissue injury. Science 354, 765-768 (2016).

33. Komada, T. et al. Macrophage uptake of necrotic cell DNA activates the AIM2 inflammasome to regulate a proinflammatory phenotype in CKD. J. Am. Soc. Nephrol. 29, 1165-1181 (2018).

34. Qi, M. et al. AIM2 promotes the development of non-small cell lung cancer by modulating mitochondrial dynamics. Oncogene 39, 2707-2723 (2020).

35. Lu, A. et al. Plasticity in PYD assembly revealed by cryo-EM structure of the PYD filament of AIM2. Cell Discov. 1, 15013 (2015).

36. Lu, A., Kabaleeswaran, V., Fu, T., Magupalli, V. G. \& Wu, H. Crystal structure of the F27G AIM2 PYD mutant and similarities of its self-association to DED/ DED interactions. J. Mol. Biol. 426, 1420-1427 (2014).

37. Sohn, J. \& Hur, S. Filament assemblies in foreign nucleic acid sensors. Curr Opin. Struct. Biol. 37, 134-144 (2016).

38. $\mathrm{Wu}, \mathrm{B}$. et al. Molecular imprinting as a signal-activation mechanism of the viral RNA sensor RIG-I. Mol. Cell 55, 511-523 (2014)

39. David, L. et al. Assembly mechanism of the CARMA1-BCL10-MALT1TRAF6 signalosome. Proc. Natl Acad. Sci. USA 115, 1499-1504 (2018).

40. Gray, J. J. et al. Protein-protein docking with simultaneous optimization of rigid-body displacement and side-chain conformations. J. Mol. Biol. 331 , 281-299 (2003).

41. Stranges, P. B. \& Kuhlman, B. A comparison of successful and failed protein interface designs highlights the challenges of designing buried hydrogen bonds. Protein Sci. 22, 74-82 (2013).

42. Chaudhury, S., Lyskov, S. \& Gray, J. J. PyRosetta: a script-based interface for implementing molecular modeling algorithms using Rosetta. Bioinformatics 26, 689-691 (2010)

43. Schmidt, F. I. et al. A single domain antibody fragment that recognizes the adaptor ASC defines the role of ASC domains in inflammasome assembly. $J$. Exp. Med. 213, 771-790 (2016).

44. Katsuno, H. et al. Actin migration driven by directional assembly and disassembly of membrane-anchored actin filaments. Cell Rep. 12, 648-660 (2015).

45. Cai, X. et al. Prion-like polymerization underlies signal transduction in antiviral immune defense and inflammasome activation. Cell 156, 1207-1222 (2014).

46. Zheng, S. Q. et al. MotionCor2: anisotropic correction of beam-induced motion for improved cryo-electron microscopy. Nat. Methods 14, 331-332 (2017).

47. Mindell, J. A. \& Grigorieff, N. Accurate determination of local defocus and specimen tilt in electron microscopy. J. Struct. Biol. 142, 334-347 (2003).

48. Tang, G. et al. EMAN2: an extensible image processing suite for electron microscopy. J. Struct. Biol. 157, 38-46 (2007).

49. Frank, J. et al. SPIDER and WEB: processing and visualization of images in 3D electron microscopy and related fields. J. Struct. Biol. 116, 190-199 (1996).

50. Pettersen, E. F. et al. UCSF Chimera-a visualization system for exploratory research and analysis. J. Comp.ut Chem. 25, 1605-1612 (2004).

51. Emsley, P., Lohkamp, B., Scott, W. G. \& Cowtan, K. Features and development of Coot. Acta Crystallogr. D Biol. Crystallogr. 66, 486-501 (2010).

52. Wang, R. Y. et al. Automated structure refinement of macromolecular assemblies from cryo-EM maps using Rosetta. Elife 5, e17219 (2016).

53. Adams, P. D. et al. PHENIX: a comprehensive Python-based system for macromolecular structure solution. Acta Crystallogr. D Biol. Crystallogr. 66, 213-221 (2010) 
54. Chen, V. B. et al. MolProbity: all-atom structure validation for macromolecular crystallography. Acta Crystallogr. D Biol. Crystallogr. 66, 12-21 (2010).

55. Lyskov, S. \& Gray, J. J. The RosettaDock server for local protein-protein docking. Nucleic Acids Res. 36, W233-W238 (2008).

56. Huang, J. \& MacKerell, A. D. Jr. CHARMM36 all-atom additive protein force field: validation based on comparison to NMR data. J. Comput. Chem. 34, 2135-2145 (2013).

57. Jo, S., Kim, T., Iyer, V. G. \& Im, W. CHARMM-GUI: a web-based graphical user interface for CHARMM. J. Comput. Chem. 29, 1859-1865 (2008).

58. Qi, Y. et al. CHARMM-GUI martini maker for coarse-grained simulations with the martini force field. J. Chem. Theory Comput. 11, 4486-4494 (2015).

59. Morriss, G. P. \& Dettmann, C. P. Thermostats: analysis and application. Chaos 8, 321-336 (1998).

60. Sagui, C. \& Darden, T. A. Molecular dynamics simulations of biomolecules: long-range electrostatic effects. Annu, Rev. Biophys. Biomol. Struct. 28, 155-179 (1999).

61. Hess, B. P-LINCS: a parallel linear constraint solver for molecular simulation. J. Chem. Theory Comput. 4, 116-122 (2008).

62. Bussi, G. \& Tribello, G. A. Analyzing and biasing simulations with PLUMED. Methods Mol. Biol. 2022, 529-578 (2019).

63. Mazanek, Z. \& Sohn, J. Tracking the polymerization of DNA sensors and inflammasomes using FRET. Methods Enzymol. 625, 87-94 (2019).

64. Matyszewski, M. \& Sohn, J. Preparation of filamentous proteins for electron microscopy visualization and reconstruction. Methods Enzymol. 625, 167-176 (2019).

65. Matyszewski, M. et al. Distinct axial and lateral interactions within homologous filaments dictate the signaling specificity and order of the AIM2ASC inflammasome. Nature Commun. Github https://doi.org/10.5281/ zenodo.4667936 (2021)

\section{Acknowledgements}

We thank Drs. Jeffry Gray and L. Mario Amzel for discussion, and Drs. Brendan Antiochos and Shuai Wu for cell culture experiments. This work was supported by American Cancer Society Research Scholars Grant (RSG-15-224-01DMC), NSF CAREER award (MCB1845003), and NIH R01GM129342 to J.S., NIH R35GM122510 to E.H.E. Computational resources were provided by Maryland Advanced Research Computing Center at Johns Hopkins University.

\section{Author contributions}

M.M. and J.S. conceived the project and designed experiments. M.M., W.Z., Z.M., J.L., and N.M. performed experiments. M.M., W.Z., Z.M., J.L., N.M., A.Y.L., E.H.E., and J.S interpreted data. M.M., W.Z., N.M., and J.S. wrote the paper which other authors commented on.

\section{Competing interests}

The authors declare no competing interests.

\section{Additional information}

Supplementary information The online version contains supplementary material available at https://doi.org/10.1038/s41467-021-23045-8.

Correspondence and requests for materials should be addressed to J.S.

Peer review information Nature Communications thanks Qian Yin and the other, anonymous, reviewer(s) for their contribution to the peer review of this work.

Reprints and permission information is available at http://www.nature.com/reprints

Publisher's note Springer Nature remains neutral with regard to jurisdictional claims in published maps and institutional affiliations.

\begin{abstract}
(c) Open Access This article is licensed under a Creative Commons Attribution 4.0 International License, which permits use, sharing, adaptation, distribution and reproduction in any medium or format, as long as you give appropriate credit to the original author(s) and the source, provide a link to the Creative Commons license, and indicate if changes were made. The images or other third party material in this article are included in the article's Creative Commons license, unless indicated otherwise in a credit line to the material. If material is not included in the article's Creative Commons license and your intended use is not permitted by statutory regulation or exceeds the permitted use, you will need to obtain permission directly from the copyright holder. To view a copy of this license, visit http://creativecommons.org/ licenses/by/4.0/.
\end{abstract}

(C) The Author(s) 2021 